\title{
Une minoration du minimum essentiel sur les variétés abéliennes
}

\author{
Aurélien Galateau
}

Résumé. On étend à la codimension générale la minoration du minimum essentiel sur les variétés abéliennes obtenue dans [Gal08], sous une conjecture concernant leurs idéaux premiers ordinaires. Cette minoration est optimale "à $\varepsilon$ près" en le degré de la sous-variété et rend inconditionnelle la démonstration, par Viada, de la conjecture de Zilber-Pink pour une courbe plongée dans une puissance de courbe elliptique.

Abstract. We extend to the general codimension the lower bound for the essential minimum on abelian varieties found in [Gal08], under a conjecture about ordinary primes in abelian varieties. This lower bound is the best expected, "up to an $\varepsilon$ ", in the degree of the subvariety and completes Viada's proof of the Zilber-Pink conjecture for a curve embedded in a power of an elliptic curve.

Classification mathématique par sujets (2010). 11G10, 11J81, 14G40.

Mots clés. Bogomolov, variété abelienne, minoration, hauteur.

Keywords. Bogomolov, abelian variety, lower bound, height.

\section{Introduction}

On poursuit dans ce travail la minoration de la hauteur normalisée sur les variétés abéliennes commencée dans [Ga108], dont on généralise le résultat principal en codimension quelconque. Commençons par rappeler les origines de notre problème. Soit $C$ une courbe algébrique de genre $g \geq 2$ définie sur $\overline{\mathbb{Q}}$ et plongée canoniquement dans sa jacobienne $J(C)$. On note $\mathscr{L}$ le fibré en droites associé et $\hat{h}_{\mathscr{L}}$ la hauteur de Néron-Tate sur $J(C)$. En 1981, Bogomolov conjecture :

Théorème A (Ullmo). Il existe $\varepsilon>0$ tel que $\left\{x \in C(\overline{\mathbb{Q}}), \hat{h}_{\mathscr{L}}(x) \leq \varepsilon\right\}$ soit fini.

Plus généralement, soit $V$ une sous-variété d'une variété abélienne $A$ munie d'un fibré en droites $\mathscr{L}$ ample et symétrique. Ici et dans la suite de l'article, les variétés considérées sont définies sur $\overline{\mathbb{Q}}$ et irréductibles. On note $\hat{h}_{\mathscr{L}}$ la hauteur de Néron-Tate associée à ce fibré. On dit que $V$ est de torsion si $V$ est la translaté d'une sous-variété abélienne par un point de torsion. Une courbe algébrique de torsion est en particulier de genre 1. On définit aussi, pour $V$ un fermé de Zariski inclus dans $A$ : 
Définition 1.1. Le minimum essentiel de $V$ est

$$
\hat{\mu}_{\mathscr{L}}^{\text {ess }}(V)=\inf \left\{\theta>0, \overline{V(\theta)}^{Z}=V(\overline{\mathbb{Q}})\right\},
$$

où $V(\theta)=\left\{x \in V(\overline{\mathbb{Q}}), \hat{h}_{\mathscr{L}}(x) \leq \theta\right\}$ et $\overline{V(\theta)} Z$ est son adhérence de Zariski.

On peut alors étendre aux sous-variétés des variétés abéliennes le théorème $\mathrm{A}$ :

Théorème $\mathbf{B}$ (Zhang). Soit $V$ une sous-variété stricte d'une variété abélienne A (ie., $V \subsetneq A$ ). Le minimum essentiel de $V$ est nul si et seulement si $V$ est de torsion.

Le résultat analogue est vrai si on remplace $A$ par un tore ( $c f$. [Zha92]) ou plus généralement par une variété semi-abélienne ( $c f$. [DP00]).

1.1. Versions quantitatives du problème de Bogomolov. Ces conjectures étant démontrées, on peut s'intéresser à une version quantitative, en précisant $\varepsilon$ dans le théorème $\mathrm{A}$ ou en minorant le minimum essentiel d'une variété qui n'est pas de torsion. Grâce au théorème des minima successifs démontré par Zhang (dans [Zha95]), ceci revient à minorer la hauteur canonique d'une telle variété. Depuis les travaux de Bombieri et Zannier (voir [BZ95] pour le cas torique et [BZ96] pour le cas abélien), on sait qu'on peut espérer obtenir une borne "uniforme" pour le minimum essentiel, ne dépendant que du degré de $V$ et de la variété abélienne $A$. Remarquons que pour obtenir de telles minorations, on doit exclure les translatés de sous-variétés abéliennes par des points qui ne sont pas de torsion. En effet, si $V=x+B \subsetneq A$, le minimum essentiel est relié à la hauteur de la projection de $x$ dans le quotient $A / B$ (pour plus de détails, voir [Lit99]) et on peut le faire tendre vers 0 en fixant le degré et la dimension de $V$.

On définit d'abord un invariant plus fin que le degré, approprié aux techniques diophantiennes :

Définition 1.2. Soit $V$ un fermé de Zariski strict de $S$ une variété semi abélienne munie d'un fibré en droites ample $\mathscr{L}$. On appelle indice d'obstruction de $V$, noté $\omega_{\mathscr{L}}(V)$,

$$
\omega_{\mathscr{L}}(V)=\inf \left\{\operatorname{deg}_{\mathscr{L}}(Z)\right\},
$$

où l'infimum est pris sur l'ensemble des hypersurfaces (non nécessairement irréductibles) de $S$ contenant $V$.

Remarque. Suivant notamment [DH00], on pourrait définir l'indice d'obstruction de $V$ comme l'infimum des $\operatorname{deg}_{\mathscr{L}}(Z)^{1 / \operatorname{codim}(Z)}$ sur les fermés $Z$ de $S$ contenant $V$. Ce choix est plus fin que celui de la définition 1.2, et les méthodes diophantiennes utilisées ici le font intervenir naturellement. Cependant, la descente finale employée dans notre 
article impose de travailler avec des hypersurfaces; de plus, on est amené à définir dans cette descente un indice d'obstruction plus compliqué qu'on compare à l'indice d'obstruction initial, ce qu'on ne peut faire de façon optimale qu'en codimension 1. En vertu d'un théorème de Chardin (corollaire 2.2 de [Cha88]), les théorèmes $\mathrm{C}$ et 1.1 ci-dessous sont encore vrais avec cette définition alternative.

Dans [AD03], Amoroso et David donnent une majoration quasi-optimale en l'indice d'obstruction d'une sous-variété $V$ d'un tore. Dans un schéma d'approximation diophantienne, ils utilisent les propriétés de ramification des corps cyclotomiques, qu'ils traduisent en un résultat métrique leur permettant d'extrapoler une fonction auxiliaire nulle sur $V$. Par le plongement standard $\mathbb{G}_{m}^{n} \hookrightarrow \mathbb{P}^{n}$ ( $\operatorname{sur} \overline{\mathbb{Q}}$ ), dont le fibré en droites associé est noté $\mathscr{L}$, on dispose d'une hauteur $h_{\mathscr{L}}$ sur les points de $\mathbb{G}_{m}^{n}$, et d'un minimum essentiel $\hat{\mu}_{\mathscr{L}}^{\text {ess }}$ sur les sous-variétés de $\mathbb{G}_{m}^{n}$. Ils démontrent alors :

Théorème C. Soit $V$ une sous-variété stricte de $\mathbb{G}_{m}^{n}$ de codimension $k$ qui n'est contenue dans aucun translaté d'un sous-tore strict de $\mathbb{G}_{m}^{n}$. On a

$$
\hat{\mu}_{\mathscr{L}}^{\mathrm{ess}}(V) \geq \frac{c(n)}{\omega_{\mathscr{L}}(V)} \times\left(\log \left(3 \omega_{\mathscr{L}}(V)\right)\right)^{-\lambda(k)},
$$

où c(n) est un réel strictement positif et $\lambda(k)=\left(9(3 k)^{(k+1)}\right)^{k}$.

Ce théorème est optimal aux termes logarithmiques près (voir la conjecture 1.2 de [AD03], et la remarque qui suit), comme le résultat de Dobrowolski ( $c f$. [Dob79]) sur le problème de Lehmer et ses généralisations.

Dans le cas des variétés abéliennes, on dispose déjà de résultats quantitatifs, mais la dépendance en le degré est moins bonne. On cite ici une version faible du théorème principal de [DP02], obtenue en comparant le rayon d'injectivité à la hauteur de $A$ suivant le lemme 6.8 de cette même référence :

Théorème D. Soit $A$ une variété abélienne de dimension $g \geq 2$ définie sur $\overline{\mathbb{Q}}$, principalement polarisée par un fibré en droites $\mathscr{L}$ ample et symétrique, et $V$ une sous-variété de A qui n'est pas translaté d'une sous-variété abélienne. Il existe alors une fonction explicite et strictement positive $C(g)$ telle que

$$
\hat{\mu}_{\mathscr{L}}^{\mathrm{ess}}(V) \geq \frac{C(g)}{d^{g+1} \max \left\{1, h_{\mathscr{L}}(A)\right\}^{g+1} \operatorname{deg}_{\mathscr{L}}(V)^{2(g+1)^{2}}},
$$

où d est le degré d'un corps de définition de $A$ et $h_{\mathscr{L}}(A)$ est la hauteur projective de l'origine de A dans le plongement associé à $\mathscr{L}^{\otimes 16}$.

Cette minoration est monomiale inverse en le degré, alors que dans le cas torique, elle est linéaire inverse en l'indice d'obstruction (aux termes logarithmiques 
près), ce qui, par le corollaire 2 de [Cha88] 2, correspond à une minoration en $\operatorname{deg}_{\mathscr{L}}(V)^{-1 / \operatorname{codim}(V)}$. Remarquons enfin que l'hypothèse du théorème $\mathrm{D}(V$ n'est pas un translaté de sous-variété abélienne stricte) est plus faible que son analogue torique dans le théorème $\mathrm{C}$ ( $V$ n'est pas incluse dans un translaté de sous-tore strict), et que cette différence se ressent dès qu'on obtient des résultats comparables au théorème $\mathrm{C}$.

1.2. Résultats et applications. Soit $A$ une variété abélienne définie sur $K$ un corps de nombres, munie d'un fibré en droites $\mathscr{L}$ ample et symétrique. Pour $\mathcal{A}$ un modèle entier de $A \operatorname{sur} \mathcal{O}_{K}$, on commence par poser l'hypothèse suivante :

Hypothèse $\mathbf{H}$. Il existe une densité positive de premiers $\mathfrak{p}$ de $\mathcal{O}_{K}$ en lesquels le $p$-rang de la fibre spéciale $\mathcal{A}_{\mathfrak{p}}$ est égal à $g$.

Sous cette hypothèse, on a démontré dans [Gal08] l'analogue du théorème $\mathrm{C}$ en petite codimension $(k \leq 2)$. Le but de ce travail est de généraliser à la codimension quelconque ce résultat. Suivant une idée nouvelle d'Amoroso (voir [Amo07]), on modifie les arguments de transcendance, ce qui permet d'insérer un principe de tiroirs dans la combinatoire du lemme de zéros. Grâce à ce raffinement, on peut mettre en place la technique de descente, ce qui n'était possible qu'en codimension 2 dans notre travail précédent. On démontre ici :

Théorème 1.1. On suppose qu'il existe un modèle entier $\mathcal{A}$ de $A$ vérifiant $H$. Soit $V$ une sous-variété stricte de codimension $k$ quelconque dans $A$. Si V n'est contenue dans aucun translaté d'une sous-variété abélienne stricte de A, on a

$$
\hat{\mu}_{\mathscr{L}}^{\text {ess }}(V) \geq \frac{C_{\mathscr{L}}(A)}{\omega_{\mathscr{L}}(V)} \times\left(\log \left(3 \operatorname{deg}_{\mathscr{L}}(V)\right)\right)^{-\lambda(k)},
$$

où $C_{\mathscr{L}}(A)$ est un réel strictement positif dépendant de $(A, \mathscr{L})$ et $\lambda(k)=(5 g(k+$ 1) $)^{k+1}$.

Remarque. - La constante $\lambda(k)$ obtenue avec ce nouveau schéma de preuve est sensiblement meilleure que celle du théorème $\mathrm{C}$.

- La minoration obtenue est optimale aux facteurs logarithmiques près. En effet, si on considère $[p]^{-1} V$, pour $V$ non incluse dans une sous-variété de torsion et $p$ un nombre premier tel que $[p]^{-1} V$ soit irréductible (cette condition est réalisée sauf pour un ensemble fini de premiers), on a $\operatorname{deg}_{\mathscr{L}}\left([p]^{-1} V\right)=p^{2 \operatorname{codim}(V)} \operatorname{deg}_{\mathscr{L}}(V) \rightarrow \infty$, alors que

$$
\hat{\mu}_{\mathscr{L}}^{\mathrm{ess}}\left([p]^{-1} V\right) \omega_{\mathscr{L}}\left([p]^{-1} V\right) \leq \hat{\mu}_{\mathscr{L}}^{\mathrm{ess}}(V) \omega \mathscr{L}(V) .
$$

- Les difficultés rencontrées dans la mise en œuvre de la descente (qu'on doit ici initialiser avec la variété $V$ plutôt qu'avec une hypersurface $Z$ réalisant $\omega_{\mathscr{L}}(V)$,

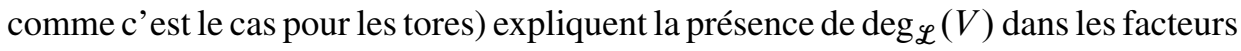
logarithmiques. 
Par un argument de projection (voir la démonstration du corollaire 5 dans [Rat04], qui s'adapte à l'identique ici), on déduit du théorème 1.1 :

Corollaire 1.2. S'il existe un modèle entier A de A vérifiant $H$, pour toute sousvariété $V$ stricte de A qui n'est pas de torsion, on a

$$
\hat{\mu}_{\mathscr{L}}^{\mathrm{ess}}(V) \geq \frac{C_{\mathscr{L}}^{\prime}(A)}{\operatorname{deg}_{\mathscr{L}}(V)^{1 /(s-\operatorname{dim}(V))}} \times\left(\log \left(3 \operatorname{deg}_{\mathscr{L}}(V)\right)\right)^{-\lambda(s-\operatorname{dim}(V))},
$$

où $x+B$ est la plus petite sous-variété de torsion contenant $V$, s est la dimension de $B$ et $C_{\mathscr{L}}^{\prime}(A)$ est un réel positif qui ne dépend que de $(A, \mathscr{L})$.

En appliquant l'inégalité des minima successifs (voir infra, 2.2 pour cette inégalité et pour la définition de $\hat{h}$ sur les sous-variétés de $A$ ), on a aussi :

Corollaire 1.3. S'il existe un modèle entier de A vérifiant l'hypothèse $\mathrm{H}$, pour toute sous-variété $V$ stricte et irréductible de codimension $k \geq 2$ dans A qui n'est contenue dans aucun translaté d'une sous-variété abélienne stricte de A, on a

$$
\hat{h}_{\mathscr{L}}(V) \geq C_{\mathscr{L}}^{\prime \prime}(A)
$$

où $C_{\mathscr{L}}^{\prime \prime}(A)$ est un réel strictement positif dépendant de $(A, \mathscr{L})$.

La seule restriction qui empêche d'obtenir une minoration inconditionnelle est donc l'hypothèse H. Elle est en fait l'objet de la conjecture suivante (voir [Pin98], § 7) :

Conjecture E. Pour toute variété abélienne A définie sur $\overline{\mathbb{Q}}$, il existe un corps de définition $K$ de $A$ et un modèle entier Â de $A$ sur $\mathcal{O}_{K}$ vérifiant l'hypothèse $H$.

Sous cette conjecture, la minoration du théorème 1.1 est vérifiée pour toutes les variétés abéliennes définies sur $\overline{\mathbb{Q}}$. En dimension 1 , pour une courbe elliptique $E$, le résultat est connu (voir [Ser68], chapitre IV, 13 et la théorie de la multiplication complexe). La validité de l'hypothèse $\mathrm{H}$ a été étendue aux surfaces abéliennes par les travaux de Katz et Ogus ( $c f$. [Ogu82], §2.7, en remarquant par le théorème de Chebotarev que les premiers de degré 1 ont une densité positive). L'hypothèse $\mathrm{H}$ est encore vraie pour les variétés abéliennes à multiplications complexes (voir [BS04], partie 5). Des conditions suffisantes, portant sur les groupes de monodromie $G_{l}$ (associés à chaque nombre premier $l$ ) de la variété abélienne, ont été données par Noot (voir [Noo95],§ 2) puis Pink ([Pin98], § 7).

Une telle minoration du minimum essentiel s'applique au problème de l'intersection d'une variété avec des sous-groupes algébriques, en direction de conjectures formulées de façon indépendante par Zilber sur les variétés semi-abéliennes, et Pink 
sur les variétés de Shimura mixtes (voir [Zil02] et [Pin05], conjectures 1.2 et 1.3). Pour $S$ un sous-ensemble de $A(\overline{\mathbb{Q}})$, on note

$$
S_{\varepsilon}=\left\{x+y, x \in S, y \in A(\overline{\mathbb{Q}}), \hat{h}_{\mathscr{L}}(y) \leq \varepsilon\right\},
$$

ainsi que

$$
\mathscr{H}=\bigcup_{\operatorname{codim} H \geq 2} H,
$$

la réunion portant sur tous les sous-groupes algébriques de $A$ (éventuellement réductibles) ayant la codimension prescrite. On garde les mêmes définitions avec le tore $\mathbb{G}_{m}^{n}$ (défini sur $\overline{\mathbb{Q}}$ ) à la place de $A$. En utilisant le théorème $\mathrm{C}$, Habegger a démontré le résultat suivant (voir [Hab08]) :

Théorème $\mathbf{F}$. Soit $C$ une courbe dans $\mathbb{G}_{m}^{n}$ qui n'est pas incluse dans le translaté d'un sous-tore strict. Alors il existe $\varepsilon>0$ tel que $C(\overline{\mathbb{Q}}) \cap \mathscr{H}_{\varepsilon}$ est fini.

Ce théorème a permis à Maurin de démontrer la conjecture de Zilber pour une courbe plongée dans un tore ( $c f$. [Mau08]) :

Théorème G. Soit $C$ une courbe algébrique irréductible de $\mathbb{G}_{m}^{n}$ non incluse dans un sous-groupe algébrique strict (éventuellement réductible). Alors $C(\overline{\mathbb{Q}}) \cap \mathcal{H}$ est fini.

Il améliore ainsi le résultat principal de l'article fondateur de Bombieri, Masser et Zannier ( $c f$. [BMZ99]). Dans le cadre abélien, des estimations de type Lehmer sur les points de petite hauteur permettent de traiter le cas des variétés abéliennes à multiplication complexe (voir [Via03], [RV03], [Rat08] et [Car08]).

Soit $E$ une courbe elliptique définie sur $\overline{\mathbb{Q}}, g \geq 2$ un entier et $\Gamma$ un sous-groupe de $E^{g}$ de rang fini. Le théorème 1.1 rend désormais inconditionnel le résultat de Viada (cf. [Via08a]) :

Théorème H. Soit $C$ une courbe algébrique incluse dans $E^{g}$.

- Si C n'est pas incluse dans un sous-groupe algébrique strict, il existe $\varepsilon>0$ tel que $C(\overline{\mathbb{Q}}) \cap \mathscr{H}_{\varepsilon}$ soit fini.

- Si C n'est pas incluse dans un translaté de sous-variété abélienne stricte, il existe $\varepsilon>0$ tel que $C(\overline{\mathbb{Q}}) \cap\left(\mathscr{H}+\Gamma_{\varepsilon}\right)$ soit fini.

Le premier point de ce théorème implique la conjecture de Zilber pour les courbes plongées dans une puissance de courbe elliptique. En raffinant son argument, Viada a depuis obtenu des résultats pour une sous-variété générale d'une puissance de courbe elliptique (voir [Via08b]). 
1.3. Plan de l'article. La preuve du théorème 1.1 est une adaptation du nouveau schéma de transcendance utilisé par Amoroso dans [Amo07]. Elle est écrite dans le formalisme classique de la géométrie diophantienne. La deuxième partie rassemble quelques rappels sur les variétés abéliennes. On y introduit notamment le plongement étiré, qui permet de négliger la constante de comparaison entre la hauteur projective et la hauteur de Néron-Tate. On construit aussi un bon ensemble de premiers de densité positive.

Dans la troisième partie, on choisit une base de dérivations adaptée, puis on démontre un lemme clé $p$-adique sur les points de torsion des variétés abéliennes. On transporte ensuite cette propriété à un polynôme en les coordonnées affines de la variétés abélienne. Dans la partie suivante, on en déduit une inégalité entre les fonctions de Hilbert d'un fermé avec multiplicité, et de ce même fermé translaté par un sous-groupe de torsion bien choisi, sans la multiplicité. Par un bon choix de paramètres, on trouve une section non triviale nulle sur ce translaté. L'application couplée d'un lemme de zéros de Philippon et d'un principe de tiroirs nous donne une inégalité concernant le degré d'un fermé obstructeur.

Si cette inégalité ne nous permet pas encore de conclure, elle amorce la phase de descente qui constitue la cinquième partie de ce travail. Cette technique est classique dans les travaux diophantiens visant à minorer les hauteurs, mais le cas des variétés abéliennes est plus difficile, en l'absence d'un relèvement du Frobenius en caractéristique nulle. On a cherché ici à "simuler combinatoirement" le Frobenius, ce qui explique qu'on travaille, dès la transcendance, avec des fermés de Zariski non nécessairement irréductibles.

Remerciements. Je tiens à remercier Sinnou David pour son aide constante pendant que je travaillais à ce problème, et le rapporteur dont les remarques m'ont permis d'améliorer significativement la rédaction de cet article.

\section{Préliminaires sur les variétés abéliennes}

Commençons par quelques rappels concernant la hauteur et le plongement étiré sur les variétés abéliennes. On fixe pour toute la suite une variété abélienne $A$ munie de $\mathscr{L}$ un fibré en droites ample et symétrique, et définie sur $K$ un corps de nombres. On suppose que $A$ vérifie l'hypothèse $\mathrm{H}$ et on fixe $\mathcal{A}$ un modèle entier de $A$ sur $\mathcal{O}_{K}$ tel que pour une densité positive d'idéaux premiers $v$ de $\mathcal{O}_{K}$, la fibre spéciale $\mathcal{A}_{v}$ soit ordinaire.

2.1. Choix de la constante. La minoration que l'on a en vue fait intervenir une constante dépendant de la variété abélienne $A$, de $\mathscr{L}$, mais aussi d'un certain nombre de choix portant sur $(A, \mathscr{L})$ : une base de $H^{0}(A, \mathscr{L})$, une base du tangent de $A$ en 0 , 
un modèle entier $\mathcal{A}$ donné par l'hypothèse $\mathrm{H}$. Par abus de langage, on dira qu'une telle constante ne dépend que de $A$.

On introduit d'abord des constantes $c_{1}, \ldots, c_{15}$ ne dépendant que de $A$, puis on choisit une constante $C_{0}$, ne dépendant que de $A$, grande devant les constantes $c_{1}, \ldots, c_{15}$ dans un sens que le cours de la preuve rendra explicitable. La constante $C_{\mathscr{L}}(A)$ du théorème 1.1 s'exprimera alors simplement en fonction de $C_{0}$ :

$$
C_{\mathscr{L}}(A):=C_{0}^{-2 \lambda(g)},
$$

la dimension $g$ pouvant même être remplacée par la codimension $k$ de $V$. Toutes ces constantes sont calculables mais notre méthode ne permet pas d'améliorer la dépendance en la hauteur de $A$ du théorème $\mathrm{D}$. En effet, la constante $C_{0}$ est déjà au mieux monomiale en $h_{\mathscr{L}}(A)$ et le choix de la fonction $\lambda$ donne un exposant particulièrement mauvais pour $h_{\mathscr{L}}(A)$.

2.2. Quelques définitions. Soit $n$ un entier positif, $K$ un corps de nombres et $x:=$ $\left[x_{0}: \cdots: x_{n}\right]$ un point de $\mathbb{P}^{n}(K)$. On définit la hauteur projective de $x$ par la formule suivante :

$$
h(x):=\frac{1}{[K: \mathbb{Q}]} \sum_{v \in M(K)} n_{v} \log \left(\max \left\{\left|x_{0}\right|_{v}, \ldots,\left|x_{n}\right|_{v}\right\}\right),
$$

où $M(K)$ désigne l'ensemble des places de $K$. Les $n_{v}$ et les valeurs absolues sont choisis de telle sorte que la formule du produit soit satisfaite :

$$
\forall y \in K, y \neq 0: \prod_{v \in M(K)}|y|_{v}^{n_{v}}=1,
$$

et que, pour tout $p$ nombre premier et $v \mid p$ on a $|p|_{v}=p^{-1}$. La formule donnant la hauteur ne dépend pas du choix d'un corps de définition $K$ de $x$, ni du choix d'un représentant de $x$ (par la formule du produit).

On a fixé une variété abélienne $A$ munie de $\mathscr{L}$, un fibré en droites ample et symétrique, et définie sur $K$, un corps de nombres. Quitte à considérer $\mathscr{L}^{\otimes 3}$, on peut supposer que $\mathscr{L}$ est projectivement normal et très ample. Il définit donc un plongement de $A$ dans un espace projectif $\mathbb{P}^{n}$ et on fixe dès à présent une base de sections $\left(Y_{0}, \ldots, Y_{n}\right)$ de $\mathscr{L}$ sur $A$, ainsi qu'une base $\left(f_{1}, \ldots, f_{g}\right)$ du tangent en 0 . Via ce plongement, pour tout point $x \in A(\bar{K})$, on peut définir sa hauteur projective qu'on note $h_{\mathscr{L}}(x)$. On définit aussi sa hauteur de Néron-Tate, donnée par la formule

$$
\hat{h}_{\mathscr{L}}(x):=\lim _{n \rightarrow \infty} \frac{h_{\mathscr{L}}([n] x)}{n^{2}},
$$

où $[n]$ désigne l'isogénie de multiplication par $n$, pour $n \in \mathbb{N}^{*}$. Cette fonction hauteur vérifie les propriétés suivantes : 
- $\hat{h}_{\mathscr{L}}(x)=0$ si et seulement si $x$ est un point de torsion de $A$;

- la hauteur est quadratique :

$$
\forall x \in A(\bar{K}), n \in \mathbb{N}: \quad \hat{h}_{\mathscr{L}}([n] x)=n^{2} \hat{h}_{\mathscr{L}}(x) ;
$$

- il existe une constante $c_{1}$ ne dépendant que de $A$ telle que

$$
\forall x \in A(\bar{K}): \quad\left|h_{\mathscr{L}}(x)-\hat{h}_{\mathscr{L}}(x)\right| \leq c_{1} .
$$

Via le plongement projectif de $A$ associé à $\mathscr{L}$, on peut définir le degré relativement à $\mathscr{L}$ d'une sous-variété $V$ de $A$, qu'on note $\operatorname{deg}_{\mathscr{L}}(V)$ (voir [Har77]), puis son indice d'obstruction $\omega_{\mathscr{L}}(V)$ (voir la définition 1.2).

Soit $V$ une sous-variété de $A$. La hauteur projective de $V$ relativement à $\mathscr{L}$, notée $h_{\mathscr{L}}(V)$, est définie comme la hauteur de la forme de Chow qui lui est associée via le plongement projectif. On construit ensuite la hauteur normalisée $\hat{h}_{\mathscr{L}}(V)$ par passage à la limite :

$$
\hat{h}_{\mathscr{L}}(V)=\lim _{n \rightarrow+\infty} \frac{h_{\mathscr{L}}([n] V) \operatorname{deg}_{\mathscr{L}}(V)}{n^{2} \operatorname{deg}_{\mathscr{L}}([n] V)} .
$$

Cette limite existe et la hauteur ainsi construite généralise la hauteur de Néron-Tate pour les points de $A$ (voir [HS00], page 450, et [Phi95]). On peut aussi définir la hauteur d'une variété par l'approche arakelovienne (cf [BGS94], partie 3). Ces deux notions coïncident, si on fait le choix de la norme $L^{2}$ pour les places archimédiennes dans la définition de la hauteur projective. Le théorème des minima successifs démontré par Zhang (dans [Zha95]) implique les inégalités suivantes :

Théorème 2.1. Soit V une sous-variété de A de dimension $d$. Alors,

$$
\frac{1}{d+1} \frac{\hat{h}_{\mathscr{L}}(V)}{\operatorname{deg}_{\mathscr{L}}(V)} \leq \hat{\mu}_{\mathscr{L}}^{\text {ess }}(V) \leq \frac{\hat{h}_{\mathscr{L}}(V)}{\operatorname{deg}_{\mathscr{L}}(V)} .
$$

2.3. Le plongement étiré. On aura besoin de rendre négligeable la constante de comparaison entre hauteur projective et hauteur canonique sur $A$. On résout généralement ce problème en utilisant le plongement étiré. Soit $M$ un entier strictement positif. On rappelle que l'isogénie de multiplication par $M$ sur $A$ est notée $[M]$ et on définit $\phi_{M}$ :

$$
\begin{aligned}
& A \longrightarrow A \times A, \\
& x \longmapsto(x,[M] x),
\end{aligned}
$$

qui induit par Segre un plongement de $A$ dans un espace projectif $\mathbb{P}^{m}$, le plongement étiré.

Le plongement étiré a été utilisé pour la première fois par Laurent dans le cadre du problème de Lehmer elliptique (cf. [Lau83]). Le principe en est le suivant : les 
techniques diophantiennes utilisent la hauteur projective, et le minimum essentiel fait intervenir la hauteur de Néron-Tate associée au plongement. On sait que la différence entre ces deux hauteurs est bornée mais la hauteur de Néron-Tate, qu'on prendra proche du minimum essentiel au cours de la preuve, peut être très petite. Il y a donc une perte d'information sur la hauteur projective. Le plongement étiré multiplie la hauteur par un paramètre assez grand, qui rend négligeable la constante de comparaison.

On note $\mathcal{M}$ le fibré en droites $\pi_{1}^{*} \mathscr{L} \otimes \pi_{2}^{*} \mathscr{L}$ sur $A \times A$, où $\pi_{1}$ et $\pi_{2}$ sont les projections canoniques de $A \times A$ sur chaque facteur. Par produit tensoriel, on déduit de la base $\left(Y_{0}, \ldots, Y_{n}\right)$ de $H^{0}(A, \mathscr{L})$ une base $Z:=\left(Z_{0}, \ldots, Z_{m}\right)$ de $H^{0}(A \times A, \mathcal{M})$. On note aussi $\mathcal{M}_{\mid A}$ la restriction de $\mathcal{M}$ à $A$ via le plongement étiré. On a

$$
\mathcal{M}_{\mid A} \simeq \mathscr{L}^{\otimes\left(M^{2}+1\right)} .
$$

Cet isomorphisme suggère le lemme suivant, qui indique la variation de la hauteur et du degré par $\phi_{M}$.

Lemme 2.2. Si $x$ est un point de $A(\bar{K})$, on a

$$
\hat{h}_{\mathcal{M}}(x)=\left(M^{2}+1\right) \hat{h}_{\mathscr{L}}(x) .
$$

Si $V$ est une sous-variété de A, on a

$$
\operatorname{deg}_{\mathcal{M}}(V)=\left(M^{2}+1\right)^{\operatorname{dim}(V)} \operatorname{deg}_{\mathscr{L}}(V) .
$$

Preuve. La première partie du lemme suit de la définition de $\phi_{M}$ par quadraticité de la hauteur. La deuxième partie est démontrée, par exemple, dans [Phi95], proposition 7.

2.4. Une première sélection de nombres premiers. Pour obtenir le lemme $p$ adique crucial dans ce travail, on devra faire un certain nombre d'hypothèses sur le premier $p$ avec lequel on travaille. Les deux premières ne sont pas très restrictives et concernent les translations et dérivations sur les coordonnées projectives des variétés abéliennes.

Lemme 2.3. Il existe des formes bi-homogènes de degré $(2,2)$ sur les coordonnées projectives représentant l'addition sur A, et données par une famille finie de coefficients $\left(g_{i}\right)_{i \in I}$ telle que pour une constante $c_{2}$ ne dépendant que de A, pour tout $p \geq c_{2}$, tout premier $v \mid p$ de $\mathcal{O}_{K}$, on a

$$
\forall i \in I: \quad\left|g_{i}\right|_{v}=1 .
$$

Preuve. Voir [LR85] ou [DP02], proposition 3.7, en remarquant qu'on a choisi $\mathscr{L}$ projectivement normal. 
Un second résultat concernant les dérivations nous servira pour montrer que les coefficients du développement de Taylor d'un polynôme en les coordonnées affines sont $v$-entiers, pour les idéaux premiers $v$ considérés. On rappelle qu'on a fixé une base $\left(f_{1}, \ldots, f_{g}\right)$ du tangent de $A$ en 0 .

Lemme 2.4. Il existe des dérivations $\left(\partial_{1}, \ldots, \partial_{g}\right)$ sur $A$, définissant une base $\left(e_{1}, \ldots\right.$, $\left.e_{g}\right) d u$ tangent en 0 , telles que pour une constante $c_{3}$ ne dépendant que de A, pour tout premier $p \geq c_{3}, v \mid p$ idéal premier de $\mathcal{O}_{K}$, on a les propriétés suivantes :

- le changement de variable de $\left(f_{1}, \ldots, f_{g}\right)$ vers $\left(e_{1}, \ldots, e_{g}\right)$ est à coefficients dans $\mathcal{O}_{K}$, et l'image de $\left(e_{1}, \ldots, e_{g}\right)$ par la réduction modulo $v$ est encore une base du tangent en 0 de $\mathcal{A}_{v}$;

- pour tout système de coordonnées affines $\left(y_{1}, \ldots, y_{n}\right)$ définies sur un ouvert de $A$ (par la non-annulation d'une des sections $Y_{k}$ fixées précédemment), on a

$$
\forall 1 \leq i \leq g, 1 \leq j \leq n: \quad \partial_{i} y_{j}=\sum_{\alpha, \beta} a_{\alpha, \beta}^{i, j} y_{\alpha} y_{\beta},
$$

avec, pour tout $(\alpha, \beta, i, j), a_{\alpha, \beta}^{i, j}$ est v-entier.

Preuve. Voir [Gal08], théorème 2.6, qui résume l'argument de [Dav91], théorème 4.1, où la hauteur des coefficients est bornée explicitement en fonction de $A$ dans le cas du plongement théta. Précisons que les coefficients de la matrice de l'application induite sur le tangent en 0 par ce choix de dérivations sont dans $K$ et ont une hauteur bornée uniquement en fonction de $A$ (dans le cas du plongement théta, voir le lemme 1.4.14 de [Dav89]). Quitte à multiplier les nouveaux paramètres par un entier assez grand (borné uniquement en fonction de $A$ et du choix de coordonnées affines), on obtient une base à coefficients dans $\mathcal{O}_{K}$. Le déterminant de la matrice de passage de la nouvelle base par rapport à $\left(f_{1}, \ldots, f_{g}\right)$ est encore de hauteur bornée par une constante ne dépendant que de $A$.

Par abus de langage, on notera encore $\left(\partial_{1}, \ldots, \partial_{g}\right)$ la base de dérivations en 0 et $\left(t_{1}, \ldots, t_{g}\right)$ le système de paramètres qui lui correspond par dualité. On ne pourra travailler directement avec cette base de dérivations mais on s'y ramènera grâce à la construction suivante.

Lemme 2.5. Il existe un ensemble fini $\left(t_{1, v}, \ldots, t_{g, v}\right)_{v \in \mathcal{V}}$ de systèmes de paramètres en 0 définis sur des ouverts $\left(\mathcal{O}_{v}\right)_{v \in \mathcal{V}}$ tels que $A(\bar{K})=\bigcup_{\nu \in \mathcal{V}} \mathcal{O}_{\nu}$.

Preuve. Si $x \in A(\bar{K})$ est différent de 0 , il existe un hyperplan passant par $x$ et évitant 0 , associé à une section $s_{x} \in H^{0}(A, \mathscr{L})$, et un hyperplan évitant à la fois $x$ et 0 , défini par l'annulation d'une section $s_{x}^{\prime} \in H^{0}(A, \mathscr{L})$. En multipliant le système de paramètres $\left(t_{1}, \ldots t_{g}\right)$ par une puissance tensorielle suffisante de $s_{x} / s_{x}^{\prime}$, on obtient un 
nouveau système de paramètres en 0 défini sur un ouvert $\mathcal{O}_{x}$ contenant $x$. Le lemme suit par compacité.

Pour tout $v \in \mathcal{V}$, on définit par dualité une base $\left(\partial_{1, \nu}, \ldots, \partial_{g, v}\right)$ de dérivations en 0 . Comme $\mathcal{V}$ est fini, il existe une constante $c_{4}$ telle que pour $p \geq c_{4}$ et $w \mid p$, les coefficients de toutes les matrices de passage de $\left(\partial_{1}, \ldots, \partial_{g}\right)$ vers les $\left(\partial_{1, v}, \ldots, \partial_{g, v}\right)_{v \in \mathcal{V}}$ (qui sont définis sur une extension finie de $K$ ) soient $w$-entiers et, des coordonnées affines étant fixées sur chaque $\mathcal{O}_{\nu}$, que leurs valeurs en 0 , qui sont bien définies, soient $w$-entières. On peut maintenant définir l'ensemble de premiers avec lesquels on travaillera :

Définition 2.1. Soit $\mathcal{P}_{\mathfrak{A}}$ l'ensemble des premiers $p \geq \max \left\{c_{2}, c_{3}, c_{4}\right\}$ non ramifiés dans $\mathcal{O}_{K}$ tels qu'il existe un idéal premier $v \mid p$ de $\mathcal{O}_{K}$ pour lequel $\mathcal{A}_{v}$ a bonne réduction ordinaire. Cet ensemble est de densité positive par choix du modèle.

\section{Points de torsion et estimations $p$-adiques}

Dans cette partie, on donne des estimations $p$-adiques concernant certains points de torsion sur les variétés abéliennes. On commence par obtenir des propriétés concernant les paramètres en 0 d'un point de torsion bien choisi, puis on transfère ces propriétés à des coordonnées affines (associées à un ouvert affine contenant le point de torsion) en utilisant les propriétés des dérivations algébriques.

3.1. Dérivations. On fixe ici une base de dérivations en tout point de $A$, qui nous permettra de transporter des estimations $p$-adiques sur un système de paramètres vers des estimations portant sur les coordonnées affines. Dans la partie suivante, on aura besoin de ce contexte différentiel pour comparer la fonction de Hilbert d'un fermé de Zariski $X$ avec multiplicité et la fonction de Hilbert d'un translaté de $X$ par un sous-groupe de torsion.

On s'est donné une base de paramètres $\left(t_{1}, \ldots, t_{g}\right)$ de $A$ en l'origine, duale de la base $\left(\partial_{1}, \ldots, \partial_{g}\right)$ du tangent de $A$ en 0 . Si $x \in A$, on obtient une base de paramètres locaux en $x$ :

$$
\left(t_{1} \circ \tau_{-x}, \ldots, t_{g} \circ \tau_{-x}\right),
$$

où $\tau_{-x}$ désigne la translation par $-x$ sur $A$. Cette base de paramètres correspond à une base du tangent de $A$ en $x$, qu'on note $\left(\partial_{1, x}, \ldots, \partial_{g, x}\right)$. Pour $\boldsymbol{k}=\left(k_{1}, \ldots, k_{g}\right) \in \mathbb{N}^{g}$, on définit

$$
|\boldsymbol{k}|=\sum_{i=1}^{g} k_{i} \quad \text { et } \quad \boldsymbol{k} !=k_{1} ! \ldots k_{g} !
$$


et les “dérivées divisées" sur $A$,

$$
\partial_{x}^{\boldsymbol{k}}:=\frac{1}{\boldsymbol{k} !} \partial_{1, x}^{k_{1}} \circ \cdots \circ \partial_{g, x}^{k_{g}} .
$$

Remarquons que l'application induite par $[M]$ sur le tangent de $A$ en 0 est la multiplication par $M$; l'image de $\left(\partial_{1}, \ldots, \partial_{g}\right)$ sous la multiplication par $M$ fournit une base de dérivations de $\phi_{M}(A)$ en 0 qu'on peut donc expliciter (voir [DH00], 4 pour plus de détails); par la suite, on se ramènera systématiquement à des dérivations de $A$ en composant des sections de $\mathcal{M}_{\mid A}$ avec le plongement étiré.

Si $T \geq 1$ est un entier et $\mathfrak{B}$ est un idéal homogène définissant un fermé $X$ de $A$ dans le plongement étiré, la puissance symbolique $\mathfrak{B}^{(T)}$ de $\mathfrak{B}$ est l'idéal engendré par les polynômes $F \in \overline{\mathbb{Q}}[\boldsymbol{Z}]$ nuls sur $X$ à un ordre au moins $T$, dans le sens suivant :

$$
\forall x \in X, \forall \boldsymbol{k} \in \mathbb{N}^{g} \text { t.q. }|\boldsymbol{k}| \leq T-1: \quad \partial_{x}^{\boldsymbol{k}}\left(F \circ \phi_{M}\right)=0 .
$$

Si $v \in \mathcal{V}$, on définit de même $\partial_{x, v}^{\boldsymbol{k}}$, pour $x \in A$ et $\boldsymbol{k} \in \mathbb{N}^{g}$. Par linéarité, le fait qu'un polynôme s'annule à un ordre donné en $x$ ne dépend pas du choix de la base de dérivations en $x$.

3.2. Coordonnées affines des points de torsion sur une variété abélienne. Soit $p \in \mathcal{P}_{\mathcal{A}}$, un idéal premier $v \mid p$ de $\mathcal{O}_{K}$ tel que $\mathcal{A}_{v}$ soit ordinaire, un point de $p$-torsion $P$ de $A$ et $\left(t_{1, v}, \ldots, t_{g, v}\right)$ un système de paramètres bien défini en $P$. On a le lemme suivant :

Lemme 3.1. Si $P$ se réduit sur 0 modulo $w$, pour un idéal premier $w \mid v$ dans un corps de définition de $P$, on a

$$
\forall 1 \leq i \leq g: \quad\left|t_{i, v}(P)\right|_{w} \leq p^{-1 / p} .
$$

Preuve. Il s'agit du corollaire 2.9 de [Gal08], la seule différence étant la convention faite sur les normes $w$-adiques.

On passe maintenant d'une propriété sur les paramètres à une propriété concernant les sections globales de $\mathcal{M}_{\mid A}$ sur $A$. Soit $T$ un entier et $\alpha \in A(\bar{K})$. On suppose que le point de $p$-torsion $P$ se réduit sur 0 modulo $w$, où $w \mid v$ est une place d'un corps de définition de $P$, de $\alpha$ et des paramètres $\left(t_{1, v}, \ldots, t_{g, v}\right)$. On note $\tau_{\alpha}$ la translation par $\alpha$ et on choisit un ouvert affine $\mathcal{O}_{v}$ contenant $P$ associé à des coordonnées affines :

$$
\left(y_{1}, \ldots, y_{n}\right) \text {. }
$$

Soit aussi $F$ une section globale d'une puissance de $\mathcal{M}_{\mid A}$, à coefficients $v$-entiers sur la base de sections de $\mathcal{M}_{\mid A}$ en tout degré formée par restriction à partir de $\left(Z_{0}, \ldots, Z_{m}\right)$, par projective normalité. On suppose que $F$ est nulle en $\alpha$ à un ordre au moins $T$ :

$$
\forall \boldsymbol{k} \in \mathbb{N}^{g} \text { t.q. }|\boldsymbol{k}| \leq T-1: \quad \partial_{\alpha, v}^{\boldsymbol{k}}\left(F \circ \phi_{M}\right)=0 .
$$


Proposition 3.2. Avec le choix des coordonnées affines $\left(y_{1}, \ldots, y_{n}\right)$, on a

$$
\left|F \circ \phi_{M}(\alpha+P)\right|_{w} \leq p^{-T / p} .
$$

Preuve. Soit $\widetilde{F}:=F \circ \phi_{M}$. Par le choix de coordonnées affines,

$$
x \longmapsto \widetilde{F}(\alpha+x)
$$

est une fonction rationnelle sur $A$ bien définie en 0 . En notant $\mathfrak{A} l$ 'anneau des fonctions sur $A, \mathfrak{m}$ l'idéal maximal associé à l' origine et $\mathfrak{A}_{\mathfrak{m}}$ le localisé de $\mathfrak{A}$ en 0 , on a l'injection

$$
\mathfrak{U}_{\mathfrak{m}} \hookrightarrow \hat{\mathfrak{A}}_{\mathfrak{m}} \simeq K\left[\left[t_{1, v}, \ldots, t_{g, v}\right]\right],
$$

qui associe à une fonction régulière sur un voisinage de 0 son développement de Taylor en les paramètres $\left(t_{1, v}, \ldots, t_{g, v}\right)$. Soit $\boldsymbol{k} \in \mathbb{N}^{g}$. Commençons par démontrer que $\partial_{\alpha, v}^{\boldsymbol{k}} \widetilde{F}$, le terme d'ordre $\boldsymbol{k}$ dans le développement en série de Taylor de $\widetilde{F}$ en $\alpha$, est $w$-entier. Par construction, on a la formule

$$
\partial_{\alpha, v}^{k} \widetilde{F}=\partial_{v}^{\boldsymbol{k}}\left(\widetilde{F} \circ \tau_{\alpha}\right)=\partial_{v}^{\boldsymbol{k}}\left(F \circ \phi_{M} \circ \tau_{\alpha}\right) .
$$

On peut supposer que les coordonnées projectives de $\alpha$ sont $w$-entières (quitte à multiplier par un entier assez grand). En itérant le lemme 2.3, on voit donc que $\phi_{M}$ et $\tau_{\alpha}$ sont donnés sur les coordonnées projectives par des polynômes à coefficients $w$-entiers. Il suit que sur l'ouvert considéré, $\widetilde{F} \circ \tau_{\alpha}$ est un polynôme à coefficients $w$-entiers en les coordonnées affines :

$$
\left(y_{1}, \ldots, y_{n}\right) .
$$

Ensuite, pour tout monôme unitaire $\boldsymbol{y}^{\boldsymbol{l}}\left(\boldsymbol{l} \in \mathbb{N}^{n}\right.$ quelconque) en les coordonnées affines $\left(y_{1}, \ldots, y_{n}\right)$, les dérivées divisées $\partial^{\boldsymbol{k}}\left(\boldsymbol{y}^{\boldsymbol{l}}\right)$, pour $\boldsymbol{k} \in \mathbb{N}^{g}$, sont $w$-entières par le choix des dérivations dans le lemme 2.4, qui sont d'une part algébriques et d'autre part à coefficients $w$-entiers. Il en va de même pour les $\partial_{v}^{\boldsymbol{k}}\left(\boldsymbol{y}^{\boldsymbol{l}}\right)$ en vertu du lemme 2.5.

La série de Taylor considérée converge donc (pour la norme $w$-adique) sur la "boule unité"

$$
\left\{x, \sup _{1 \leq i \leq g}\left\{\left|t_{i, v}(x)\right|_{w}\right\}<1\right\},
$$

et par le lemme 3.1, elle converge en $P$. On a donc, dans une complétion $w$-adique,

$$
\widetilde{F}(\alpha+P)=\sum_{\boldsymbol{k} \in \mathbb{N}^{g}} \partial_{\alpha, \nu}^{\boldsymbol{k}} \widetilde{F} \cdot \boldsymbol{t}_{v}(P)^{\boldsymbol{k}} .
$$

Puisque $F$ est nulle à l'ordre $T$ en $\alpha$, la proposition suit du lemme 3.1, de la $w$-intégrité des coefficients de cette série formelle, et de l'inégalité ultramétrique. 


\section{Section nulle sur une réunion de translatés de la variété}

Suivant la stratégie de [Amo07], on utilise la propriété $p$-adique démontrée dans le paragraphe précédent de façon "équivariante", dans le but de comparer deux fonctions de Hilbert, celle d'un fermé $X$ avec multiplicité $T$, et celle du translaté de $X$ par un sous-groupe de torsion sans multiplicité. Cette méthode donne directement l'existence d'une section nulle sur des translatés de $V$, alors que la précédente (voir [AD03]) commence par trouver une section nulle sur $V$ avec forte multiplicité avant d'extrapoler aux translatés. Cette approche plus "intrinsèque" simplifie la preuve de la façon suivante (ces trois points étant liés) :

- on peut se contenter de considérer les points de $p$-torsion pour un seul premier $p$;

- on n'est plus obligé d'itérer "l'extrapolation" en translatant plusieurs fois par des groupes de torsion;

- on utilise un lemme de zéros plus simple.

4.1. Le choix des paramètres. Soit $X$ un fermé de Zariski strict de $A$, d'indice d'obstruction $\omega_{\mathscr{L}}(X)$. On introduit un certain nombre de paramètres dépendant de $\omega_{\mathscr{L}}(X)$ et de $A$. On rappelle (voir 2.1) que $C_{0}$ est une constante ne dépendant que de $A$, grande devant toutes les constantes du problème (ne dépendant que de $A$ ). Soit $\Delta$ un paramètre vérifiant l'inégalité suivante :

$$
\Delta(X) \geq C_{0} \log \left(3 \omega_{\mathscr{L}}(X)\right)
$$

La présence de $C_{0}$ dans ce paramètre permettra souvent de simplifier les calculs en éliminant les constantes dans les termes ayant une dépendance logarithmique en $\omega_{\mathscr{L}}(X)$. La méthode diophantienne fournira in fine une minoration de $\hat{\mu}_{\mathscr{L}}^{\text {ess }}(V) \omega_{\mathscr{L}}(V)$ dépendant explicitement de $\Delta(V)$, pour $V$ vérifiant les hypothèses du théorème 1.1 ; on optimisera cette minoration en prenant $\Delta(V)$ de l'ordre de $\log \left(\operatorname{deg}_{\mathscr{L}}(V)\right)$.

On choisit d'abord $p \in \mathcal{P}_{\mathcal{A}}$ ayant une dépendance d'ordre logarithmique en $\omega_{\mathscr{L}}(X)$ dans le sens suivant:

$$
\log (p) \leq c_{5} \log (\Delta)
$$

où on a posé : $c_{5}:=(5 g(g+1))^{g+1}$ (ceci étant imposé par le choix du nombre premier dans le paragraphe 5.1). Soit $v \mid p$ un idéal de $\mathcal{O}_{K}$ de bonne réduction ordinaire (un tel idéal existe, par définition de $\mathcal{P}_{\mathcal{A}}$ ).

On suppose dans toute cette partie que le minimum essentiel de $X$ est majoré de la façon suivante :

$$
\hat{\mu}_{\mathscr{L}}^{\mathrm{ess}}(X)<\frac{\log (p)}{\Delta p \omega_{\mathscr{L}}(X)} .
$$


On poursuit avec l'entier $T$ définissant la multiplicité. L'argument $p$-adique qu'on va utiliser impose que $T$ soit plus grand que $\frac{\Delta p}{\log (p)}$, et l'ajustement des paramètres (pour comparer les fonctions de Hilbert, puis pour le lemme de zéros) force que $T$ soit en fait de l'ordre de ce quotient. On prend donc

$$
T:=\left[\frac{\Delta p}{\log (p)}\right] .
$$

La comparaison des "fonctions de Hilbert" fait intervenir le paramètre $L$ et impose que celui-ci soit assez petit (de l'ordre d'une constante). Le choisir assez grand permet de prendre $M^{2}$ plus petit (puisque c'est le produit $L M^{2}$ qui intervient typiquement dans la preuve), donc d'améliorer la minoration du minimum essentiel. Ceci nous incite à prendre

$$
L:=\left[C_{0}^{1 / 2}\right]
$$

Remarquons que le contexte du plongement étiré "décale" les paramètres : le choix de l'entier $L$ du cas torique correspond grosso modo à $M^{2}$ dans le cas abélien.

Cet entier $M$ doit être assez grand pour que la comparaison des "fonctions de Hilbert" considérées donne l'existence d'une section non-nulle (majoration de 1' "exposant de Dirichlet" selon la terminologie diophantienne, voir par exemple [DH00], 5.3). On doit ensuite le prendre assez petit pour obtenir une bonne majoration du degré d'un "fermé obstructeur" après utilisation du lemme de zéros :

$$
M:=\left[\left(\frac{\Delta p \omega_{\mathscr{L}}(X)}{\log (p)}\right)^{1 / 2}\right] .
$$

L'inégalité (1) permet alors que la hauteur des petits points de $X$ dans le plongement étiré soit bornée, ce qui suffit puisqu'on travaille avec la hauteur projective, dont la différence à la hauteur de Néron-Tate est bornée par une constante.

Remarque. On pourrait aussi choisir une stratégie légèrement différente, et construire une section de $\mathcal{M}$ sur $A \times A$ non-nulle sur un translaté de $X$ (ce qui est fait par exemple dans [DH00]). Avec un autre choix de paramètres, en particulier $L$ très proche de $M^{2}$, on montrerait qu'une telle section est non-nulle sur l'image de $A$ par le plongement étiré, et que la dimension de l'espace des sections sur $A \times A$ est correctement minorée. Cette méthode donne ici des résultats comparables.

4.2. Comparaison de "fonctions de Hilbert". On rappelle qu'on a fixé une base $\boldsymbol{Z}:=\left(Z_{0}, \ldots, Z_{m}\right)$ de sections de $\mathcal{M}$ sur $A \times A$ (voir 2.3). L'image par le plongement étiré du fermé $X$ est définie par un idéal homogène $\mathfrak{B} \subset \overline{\mathbb{Q}}[\boldsymbol{Z}]$. On pose

$$
\operatorname{Ker}[p]^{(v)}:=\{x \in A[p], x \equiv 0(\bmod v)\} .
$$


On note aussi pour simplifier

$$
X^{\prime}:=X+\operatorname{Ker}[p]^{(v)},
$$

et l'image de ce fermé par le plongement étiré est définie par un idéal homogène $\mathfrak{B}^{\prime}$.

Définition 4.1. Si $\mathfrak{s}$ est un idéal définissant un sous-schéma de $\phi_{M}(A)$ (non nécessairement réduit ou irréductible), on note $H(\mathfrak{I}, L)$ la dimension de l'image du morphisme de restriction :

$$
\begin{aligned}
r_{\mathfrak{I}}: H^{0}\left(A, \mathcal{M}_{\mid A}^{\otimes L}\right) & \longrightarrow[\overline{\mathbb{Q}}[\boldsymbol{Z}] / \mathfrak{I}]_{L}, \\
f & \longrightarrow[f]_{\mathfrak{I}} .
\end{aligned}
$$

La proposition suivante est l'analogue de la proposition 2.1 de [Amo07] et constitue le point clé de cette approche équivariante.

Proposition 4.1. On a l'inégalité suivante :

$$
H\left(\mathfrak{B}^{\prime}, L\right) \leq 2 H\left(\mathfrak{B}^{(T)}, L\right) .
$$

Preuve. Soit $\theta$ un réel positif tel qu'on ait

$$
\hat{\mu}_{\mathscr{L}}^{\mathrm{ess}}(X)<\theta<\frac{1}{M^{2}}
$$

ceci étant possible par (1) et choix de $M$. On commence par considérer l'ensemble

$$
X(\theta)=\left\{x \in X(\bar{K}), \hat{h}_{\mathscr{L}}(x) \leq \theta\right\} .
$$

Cet ensemble est Zariski-dense dans $X$ et l'ensemble $X^{\prime}(\theta)=X(\theta)+\operatorname{Ker}[p]^{(v)}$ est Zariski-dense dans $X^{\prime}$. Pour simplifier les notations, on pose

$$
h:=H\left(\mathfrak{B}^{\prime}, L\right) \quad \text { et } \quad h^{\prime}:=H\left(\mathfrak{B}^{\prime}, L\right)-H\left(\mathfrak{B}^{(T)}, L\right) .
$$

On peut de plus supposer que $h^{\prime} \geq 1$, la proposition étant déjà démontrée sinon. Posons

$$
S:=\operatorname{Ker}\left(r_{\mathfrak{B}^{\prime}}\right),
$$

et filtrons cet espace vectoriel. Pour tout $d \in \mathbb{N}^{*}$, on définit

$$
S_{d}:=\left\{F \in H^{0}\left(A, \mathcal{M}_{\mid A}^{\otimes L}\right), \forall x \in X^{\prime}(\theta),[K(x): K] \leq d: F(x)=0\right\} .
$$

Les $S_{d}$ forment une suite décroissante de sous-espaces vectoriels de $H^{0}\left(A, \mathcal{M}_{\mid A}^{\otimes L}\right)$ (dont la dimension est finie). L'intersection des $S_{d}$ étant égale à $S$, cette suite est stationnaire à partir d'un certain rang. On a alors, pour un entier $d_{0}$ assez grand,

$$
S_{d_{0}}=\left\{F \in S, \forall x \in X^{\prime}(\theta): F(x)=0\right\} .
$$


Comme $X^{\prime}(\theta)$ est Zariski-dense dans $X^{\prime}$, on a

$$
S=S_{d_{0}} .
$$

Les points de $X^{\prime}(\theta)$ étant définis sur une extension de degré au plus $d_{0}$ sont en nombre fini par le "théorème de Northcott". On note $\left(x_{i}\right)_{1 \leq i \leq i_{0}}$ ces points. On choisit aussi $\left(Q_{j}\right)_{1 \leq j \leq j_{0}}$, une base de $H^{0}\left(A, \mathcal{M}_{\mid A}^{\otimes L}\right)$ formée de monômes en les $Z_{j}$ de degré $L$ (le plongement étant projectivement normal). De plus, pour tout $1 \leq i \leq i_{0}$, on choisit $\alpha_{i} \in X(\theta)$ et $P_{i} \in \operatorname{Ker}[p]^{(v)}$ tels que : $x_{i}=\alpha_{i}+P_{i}$.

On note $S^{\perp}$ l'orthogonal de $S$ dans $H^{0}\left(A, \mathcal{M}_{\mid A}^{\otimes L}\right)$ pour la structure euclidienne usuelle avec le choix de la base $\left(Q_{j}\right)_{1 \leq j \leq j_{0}}$. Cet orthogonal est de dimension $h$, engendré par les $\left(Q_{j}\left(x_{i}\right)\right)_{i, j}$. On peut donc trouver $h$ points et $h$ multi-indices tels que (quitte à renuméroter)

$$
\mathfrak{D}:=\operatorname{det}\left(Q_{j}\left(x_{i}\right)\right)_{1 \leq i, j \leq h} \neq 0 .
$$

Remarquons que ce déterminant est défini dans un espace projectif par plongements de Segré et Véronese, et que son annulation a donc un sens. On peut de plus choisir un ouvert affine pour chaque $x_{i}=\alpha_{i}+P_{i}$ et on choisit l'ouvert donné par la proposition 3.2 pour le point $P_{i}$ dans les calculs qui suivent. Les $\left(Q_{j}\right)_{1 \leq j \leq h}$ sont deux-à-deux distincts et par la non-annulation du déterminant, ils engendrent un espace vectoriel de dimension $h$. On considère la projection

$$
\pi: \operatorname{Vect}\left(Q_{1}, \ldots, Q_{h}\right) \longrightarrow\left[\overline{\mathbb{Q}}[\boldsymbol{Z}] / \mathfrak{B}^{(T)}\right]_{L},
$$

qui n'est autre que la restriction de $r_{\mathfrak{B}(T)}$ à l'espace de sections considéré. Son noyau est de dimension au moins $h^{\prime}$. Ceci signifie qu'il existe $h^{\prime}$ sections en degré $L$ linéairement indépendantes :

$$
G_{k}=\sum_{j=1}^{h} g_{k, j} Q_{j}, \quad k=1, \ldots, h^{\prime},
$$

nulles sur $X$ à un ordre au moins $T$. On prend désormais $K^{\prime}$ une extension finie de $K$ sur laquelle $X$ et les coordonnées des points de $\operatorname{Ker}[p]^{(v)}$ sont définis, et $w \mid v$ une place ultramétrique de $K^{\prime}$. Quitte à faire des opérations élémentaires sur la matrice $\left(g_{k, j}\right)_{k, j}$, on peut alors supposer

$$
G_{k}=\sum_{j=1}^{h-k+1} g_{k, j} Q_{j},
$$

et, pour tout $1 \leq k \leq h^{\prime}$,

$$
\begin{array}{ll}
\left|g_{k, j}\right|_{w} \leq 1 & \text { si } j<h-k+1, \\
\left|g_{k, j}\right|_{w}=1 & \text { si } j=h-k+1 .
\end{array}
$$


En utilisant ces propriétés, on remplace les $h^{\prime}$ dernières colonnes de la matrice $\left(Q_{j}\left(x_{i}\right)\right)_{1 \leq i, j \leq h}$ par les colonnes :

$$
C_{k}:={ }^{t}\left(G_{k}\left(x_{1}\right), \ldots, G_{k}\left(x_{h}\right)\right), \quad 1 \leq k \leq h^{\prime},
$$

en substituant d'abord $C_{1}$ à la dernière colonne, puis $C_{2}$ à l'avant-dernière, et ainsi de suite. $\mathrm{Si}$ on note $\mathfrak{D}^{\prime}$ le nouveau déterminant, on a alors :

$$
\left|\mathfrak{D}^{\prime}\right|_{w}=|\mathfrak{D}|_{w}
$$

Les polynômes $G_{k}$ sont nuls à un ordre au moins $T$ en tous les points $\alpha_{i}$, pour $1 \leq i \leq h$ et ils sont à coefficients $w$-entiers. On est donc en situation d'utiliser la proposition 3.2 qui donne, pour $1 \leq i \leq h$ et $1 \leq k \leq h^{\prime}$,

$$
\left|G_{k}\left(x_{i}\right)\right|_{w} \leq p^{-\frac{T}{p}}
$$

On peut maintenant majorer $\left|\mathfrak{D}^{\prime}\right|_{w}$ en développant successivement le déterminant selon les $h^{\prime}$ dernières colonnes. Sur la $i$-ème ligne de chacune des $h-h^{\prime}$ premières colonnes, on majore la norme de chaque coefficient par

$$
\max \left\{1,\left|z_{1}\left(x_{i}\right)\right|_{w}, \ldots,\left|z_{m}\left(x_{i}\right)\right|_{w}\right\}^{L},
$$

où $\left(z_{1}, \ldots, z_{m}\right)$ est un système de coordonnées affines sur un ouvert contenant $x_{i}$. Le système de coordonnées dépend du point, mais on omettra de préciser cette dépendance puisque la majoration fera vite apparaître la hauteur, qui ne dépend pas du choix des coordonnées affines. On trouve finalement, par la formule donnant le déterminant en fonction de ses coefficients (sur les $h-h^{\prime}$ premières colonnes) et l'inégalité ultramétrique :

$$
|\mathfrak{D}|_{w}=\left|\mathfrak{D}^{\prime}\right|_{w} \leq p^{-h^{\prime} \frac{T}{p}} \prod_{i=1}^{h} \max \left\{1,\left|z_{1}\left(x_{i}\right)\right|_{w}, \ldots,\left|z_{m}\left(x_{i}\right)\right|_{w}\right\}^{L} .
$$

Si $w$ est une autre place de $K^{\prime}$, on se contente d'estimations triviales et, dans le cas archimédien, la formule des déterminants fait cette fois intervenir le facteur $h$ !. On a

$$
|\mathfrak{D}|_{w} \leq h ! \prod_{i=1}^{h} \max \left\{1,\left|z_{1}\left(x_{i}\right)\right|_{w}, \ldots,\left|z_{m}\left(x_{i}\right)\right|_{w}\right\}^{L} .
$$

On écrit la formule du produit, dans sa version logarithmique, pour le déterminant $\mathfrak{D}$ qui est défini sur le corps $K^{\prime}$ :

$$
0 \leq-\frac{h^{\prime} T}{[K: \mathbb{Q}]} \frac{\log (p)}{p}+h \log (h)+h L \max _{1 \leq i \leq h}\left\{h_{\mathcal{M}}\left(x_{i}\right)\right\} .
$$


On utilise ensuite la comparaison entre hauteur projective et hauteur de Néron-Tate (voir 2.2) et la variation de la hauteur par plongement étiré (voir le lemme 2.2) :

$$
\frac{h^{\prime} T}{[K: \mathbb{Q}]} \frac{\log (p)}{p} \leq h \log (h)+2 h L\left(M^{2} \theta+c_{1}\right) .
$$

Puis, pour une constante $c_{6}$ ne dépendant que de $A$, comme $L=\left[C_{0}^{1 / 2}\right]$ :

$$
\frac{h^{\prime}}{h} \leq c_{6} C_{0}^{1 / 2} \frac{p}{T \log (p)} \log (h) .
$$

On majore le terme $\log (h)$ en utilisant l'inclusion triviale

$$
\operatorname{Im}\left(r_{\mathfrak{B}^{\prime}}\right) \subset H^{0}\left(A, \mathcal{M}_{\mid A}^{\otimes L}\right),
$$

ce qui donne

$$
\log (h) \leq c_{7} \log \left(L M^{2}\right),
$$

pour une constante $c_{7}$ ne dépendant que de $A$ (par le théorème de Hilbert-Serre). On en déduit, par choix des paramètres (voir 4.1) et pour une constante $c_{8}$ ne dépendant que de $A$ :

$$
\begin{aligned}
\frac{h^{\prime}}{h} & \leq c_{8} C_{0}^{1 / 2} \frac{p}{T \log (p)} \log \left(L M^{2}\right) \\
& \leq c_{8} C_{0}^{1 / 2} \frac{\log \left(L M^{2}\right)}{2 \Delta} \\
& \leq C_{0}^{2 / 3} \frac{\log (\Delta)}{2 \Delta}+C_{0}^{2 / 3} \frac{\log \left(\omega_{\mathscr{L}}(X)\right)}{2 \Delta} \\
& \leq \frac{1}{2},
\end{aligned}
$$

la constante $C_{0}$ étant prise suffisamment grande. La proposition est donc entièrement démontrée, par les définitions de $h$ et $h^{\prime}$.

4.3. Utilisation du lemme de zéros. On montre qu'avec le choix des paramètres, et la comparaison des fonctions de Hilbert faite précédemment, il existe une section nulle sur $X^{\prime}$, le translaté de $X$ par un sous-groupe de torsion. Puis à l'aide d'un lemme de zéros classique de Philippon, on en déduit une inégalité impliquant le degré d'une variété obstructrice. Cette inégalité ne permet pas de conclure et on devra recourir à un argument de descente. À cette fin, on raffine le lemme de zéros avec un principe de tiroirs pour montrer que la variété obstructrice contient une bonne proportion de composantes du fermé algébrique d'origine, si celui-ci n'est plus irréductible.

Corollaire 4.2. Il existe une section de $\mathscr{L}$ sur A en degré $L\left(M^{2}+1\right)$, non nulle, qui s'annule sur $X^{\prime}$. 
Preuve. On va en fait démontrer qu'il existe une section de $\mathcal{M}_{\mid A}$ en degré $L$, non nulle sur $A$, qui s'annule sur $X^{\prime}$. Ceci impliquera le corollaire par l'isomorphisme

$$
\mathcal{M}_{\mid A} \simeq \mathscr{L}^{\otimes\left(M^{2}+1\right)} .
$$

On commence par majorer le membre de droite dans l'inégalité de la proposition 4.1. On choisit d'abord une hypersurface $Z$ contenant $X$, réalisant $\omega_{\mathscr{L}}(X)$ et associée à un idéal $\mathfrak{C}$. Suivant un calcul de géométrie différentielle classique (1' "astuce de Philippon-Waldschmidt", voir par exemple [PW88], lemme 6.7, [DH00], lemme 5.1 ou [Gal08], proposition 5.1), on obtient :

$$
H\left(\mathfrak{B}^{(T)}, L\right) \leq H\left(\mathfrak{C}^{(T)}, L\right) \leq T H(\mathfrak{C}, L) .
$$

Par le théorème de Chardin et le lemme 2.2, on a, pour une constante $c_{9}$ ne dépendant que de $A$ :

$$
H(\mathfrak{C}, L) \leq \operatorname{dim}_{\overline{\mathbb{Q}}}[\overline{\mathbb{Q}}[\boldsymbol{Z}] / \mathfrak{C}]_{L} \leq c_{9}\left(L M^{2}\right)^{g-1} \omega_{\mathscr{L}}(X) .
$$

Par ailleurs, la dimension de l'espace des sections est minorée de la façon suivante (en utilisant le théorème de Hilbert-Serre), pour $c_{10}$ ne dépendant que de $A$ :

$$
\operatorname{dim}_{\overline{\mathbb{Q}}}\left(H^{0}\left(A, \mathcal{M}_{\mid A}^{\otimes L}\right)\right) \geq c_{10}\left(L M^{2}\right)^{g} .
$$

Si toute section de $\mathcal{M}_{\mid A}$ sur $A$ en degré $L$ nulle sur $X^{\prime}$ est identiquement nulle, on a l'inégalité

$$
c_{10}\left(L M^{2}\right)^{g} \leq H\left(\mathfrak{B}^{\prime}, L\right) \leq 2 H\left(\mathfrak{B}^{(T)}, L\right) \leq 2 c_{9} T\left(L M^{2}\right)^{g-1} \omega_{\mathscr{L}}(X) .
$$

Et pour une constante $c_{11}$ ne dépendant que de $A$ :

$$
L M^{2} \leq c_{11} T \omega_{\mathscr{L}}(X) .
$$

Le choix des paramètres donne

$$
\frac{1}{4} C_{0}^{1 / 2} \frac{\Delta p \omega_{\mathscr{L}}(X)}{\log (p)} \leq c_{11} \frac{\Delta p \omega_{\mathscr{L}}(X)}{\log (p)},
$$

ce qui est absurde. Le corollaire est donc entièrement démontré.

On dit qu'un fermé $Z$ de $A$ est incomplètement défini par des formes de degré $\leq L$ si toutes les composantes de $Z$ sont des composantes de $A \cap Z(I)$, où $I$ est un idéal homogène dont l'image modulo $\mathfrak{A}$ (l'idéal de définition de $A$ ) est engendrée par des polynômes de degré $\leq L$. On a la version faible de la proposition 3.3 de [Phi86] : 
Lemme 4.3. Si $Z$ est un fermé de A de codimension $k^{\prime}$ incomplètement défini par des formes de degré $\leq L$, il existe un réel $c_{12}$ ne dépendant que de A tel que

$$
\operatorname{deg}_{\mathscr{L}}(Z)^{1 / k^{\prime}} \leq c_{12} L
$$

Ce lemme nous permet d'obtenir le résultat suivant concernant un "fermé obstructeur".

Proposition 4.4. Soit $n_{X}$ le nombre de composantes irréductibles de X. Il existe un fermé équidimensionnel $Z$ contenant au moins $m_{X}$ composantes irréductibles de $X$, où $m_{X}$ est le plus petit entier supérieur ou égal à $n_{X} / g$, tel que

$$
\operatorname{deg}_{\mathscr{L}}\left(Z+\operatorname{Ker}[p]^{(v)}\right)^{1 / k^{\prime}} \leq \frac{\Delta^{2} p \omega_{\mathscr{L}}(X)}{\log (p)} .
$$

Preuve. Le corollaire 4.2 donne l'existence d'une section non nulle $F$ de $\mathscr{L}$ en degré $L\left(M^{2}+1\right)$ nulle sur $X+\operatorname{Ker}[p]^{(v)}$. Considérons l'ensemble algébrique $W$ défini par les équations

$$
F(x+P)=0, \quad P \in \operatorname{Ker}[p]^{(v)} .
$$

On a une décomposition de $W$ en fermés équidimensionnels,

$$
W=\bigcup_{1 \leq i \leq g} W_{i},
$$

où $i$ désigne la codimension de $W_{i}$. De plus, il suit de la définition de $W$ que

$$
\forall 1 \leq i \leq g: \quad W_{i}=W_{i}+\operatorname{Ker}[p]^{(v)} .
$$

Le fermé $W$ contient $X$, donc chaque composante irréductible de $X$ est incluse dans une composante irréductible de $W$ et le principe des tiroirs de Dirichlet nous garantit qu'il existe $i_{0}$ tel que $W_{i_{0}}$ contient $m \geq m_{X}$ composantes irréductibles de $X$.

Notons $Z$ le fermé $W_{i_{0}}$ et $k^{\prime}$ sa codimension. Par le lemme 2.3, le fermé $Z$ est incomplètement défini par des équations de degré au plus $2 L\left(M^{2}+1\right)$. Le lemme 4.3 nous donne donc :

$$
\begin{aligned}
\operatorname{deg}_{\mathscr{L}}(Z)^{1 / k^{\prime}} & \leq 2 c_{12} L M^{2} \\
& \leq 2 c_{12} C_{0}^{1 / 2} \frac{\Delta p \omega_{\mathscr{L}}(X)}{\log (p)} \\
& \leq \frac{\Delta^{2} p \omega_{\mathscr{L}}(X)}{\log (p)} .
\end{aligned}
$$

Comme $Z=Z+\operatorname{Ker}[p]^{(v)}$, la proposition est entièrement démontrée. 


\section{Descente finale}

Pour finir la démonstration du théorème 1.1, on va d'abord préciser la proposition précédente, en choisissant un bon nombre premier $p$. Le résultat obtenu avec $X=V$ ne nous permet pas encore de conclure, car il manque une hypothèse de coprimalité entre la partie discrète du stabilisateur du fermé obstructeur (qui est une variété dans ce cas) et le premier $p$. On fait donc une descente dont le principe est le suivant : on itère la proposition 4.4 avec une suite de fermés construits à partir de $V$, et l'hypothèse de coprimalité en décalé ; on obtient l'égalité des dimensions entre deux crans successifs, ce qui permet alors de ramener l'hypothèse de coprimalité au même niveau et d'obtenir enfin une contradiction.

5.1. Choix du premier. On va d'abord fixer le premier $p$. Celui-ci doit avoir une dépendance logarithmique en $\omega_{\mathscr{L}}(X)$, pour que la minoration du minimum essentiel ait la précision espérée ; on a d'ailleurs eu besoin de faire cette hypothèse "raisonnable" dans le paragraphe 4.1. Dans la descente, on construira des suites de premiers de plus en plus petits et suffisamment espacés. On commence par définir deux nouveaux paramètres utiles dans la mise en place de la descente, un réel positif $\rho$ qui permet de quantifier le choix de $p$, et un entier strictement positif $R$ qui sert à fixer l'hypothèse de coprimalité dont nous aurons besoin. On fait donc les hypothèses suivantes concernant ces deux paramètres :

$$
\Delta \geq \max \left\{C_{0} \log \left(\left(3 \operatorname{deg}_{\mathscr{L}}(X)\right), \log (R)\right\} \quad \text { et } \quad 2 \leq \rho \leq(5 g(k+1))^{k+1} .\right.
$$

On rappelle que $n_{X}$ est le nombre de composantes irréductibles de $X$ et $m_{X}$ est le plus petit entier supérieur ou égal à $n_{X} / g$. On obtient la "quasi contradiction" :

Proposition 5.1. On suppose que $X$ n'est pas incluse dans le translaté d'une sousvariété abélienne et que son minimum essentiel est majoré de la façon suivante :

$$
\hat{\mu}_{\mathscr{L}}^{\mathrm{ess}}(X) \omega_{\mathscr{L}}(X)<\frac{1}{\Delta^{\rho+1}} .
$$

Alors il existe un nombre premier $p$, un idéal premier $v \mid p$ de $\mathcal{O}_{K}$ de bonne réduction ordinaire pour $A$, et un fermé équidimensionnel $Z$, de codimension $k^{\prime} \geq 1$, contenant au moins $m_{X}$ composantes de $X$, tels que

- l'entier $p$ est premier avec $R$ et

$$
\frac{\Delta^{\rho}}{2} \leq p \leq \Delta^{\rho}
$$

- on a l'inégalité

$$
\operatorname{deg}_{\mathscr{L}}\left(Z+\operatorname{Ker}[p]^{(v)}\right)^{1 / k^{\prime}}<\Delta^{2} p \omega_{\mathscr{L}}(X) .
$$


Preuve. Il s'agit essentiellement de démontrer qu'il existe un premier $p \in \mathcal{P}_{\mathcal{A}}$ premier à $R$ et vérifiant les inégalités annoncées. Notons $m_{\Delta}$ le nombre de premiers $p$ de $\mathcal{P}_{\mathcal{A}}$ vérifiant

$$
\Delta^{\rho} / 2 \leq p \leq \Delta^{\rho},
$$

et $n_{R}$ le nombre de premiers de $\mathcal{P}_{\mathcal{A}}$ divisant $R$. Les premiers de $\mathcal{P}_{\mathcal{A}}$ sont en densité $c_{13}>0$ fixée avec $A$, et comme $\Delta \geq C_{0}$, quitte à prendre cette constante assez grande, le théorème des nombres premiers nous donne

$$
m_{\Delta} \geq c_{13} \frac{\Delta^{\rho}}{3 \rho \log (\Delta)} ;
$$

de plus, on a

$$
R \geq \prod_{p \in \mathcal{P}_{\mathcal{A}}} p^{v_{p}(R)} \geq \prod_{p \in \mathcal{P}_{\mathcal{A}}} p \geq 2^{n_{R}} .
$$

On trouve donc

$$
\begin{aligned}
m_{\Delta}-n_{R} & \geq c_{13} \frac{\Delta^{\rho}}{3 \rho \log (\Delta)}-\frac{\log (R)}{\log (2)} \\
& \geq c_{13} \Delta\left(\frac{\Delta^{\rho-1}}{3 \rho \log (\Delta)}-\frac{1}{\log (2)}\right) \\
& \geq c_{13} \Delta ;
\end{aligned}
$$

car $\rho \geq 2$, et avec $C_{0}$ assez grande. Pour la même raison et comme $c_{13}>0$, on a finalement

$$
m_{\Delta}-n_{R} \geq 1 .
$$

L'existence d'un premier $p \in \mathcal{P}_{A}$ vérifiant l'encadrement prescrit et ne divisant pas $R$ est donc acquise.

Pour appliquer la proposition précédente, il reste à voir que l'hypothèse faite ici sur le minimum essentiel est plus restrictive que (1). La majoration de $p$ donne

$$
\hat{\mu}_{\mathscr{L}}^{\text {ess }}(X) \omega \mathscr{L}(X)<\frac{1}{\Delta^{\rho+1}} \leq \frac{1}{\Delta p} \leq \frac{\log (p)}{\Delta p} .
$$

On peut donc appliquer la proposition 4.4, qui donne l'existence du fermé $Z$ vérifiant les propriétés annoncées.

Remarque. Expliquons maintenant l'idée qui va guider la fin de la preuve. On prend $X=V$ une variété irréductible qui n'est pas incluse dans le translaté d'une sousvariété abélienne stricte de $A$. Une version simplifiée de la procédure diophantienne employée jusqu'ici donne, pour $V$ irréductible, l'existence d'un fermé obstructeur $Z$ lui aussi irréductible de codimension $k^{\prime} \geq 1$ contenant un translaté de $V$, et 
d'un premier $p$ vérifiant les inégalités de la proposition 5.1. On aimerait imposer l'hypothèse

$$
p \nmid\left[\operatorname{Stab}(Z): \operatorname{Stab}(Z)^{0}\right],
$$

où $\operatorname{Stab}(Z)$ désigne le stabilisateur de $Z$, et $\operatorname{Stab}(Z)^{0}$ la composante connexe de $\operatorname{Stab}(Z)$ contenant l'origine. Dans ce cas, le groupe quotient $\operatorname{Stab}(Z) / \operatorname{Stab}(Z)^{0}$, qui se relève dans $\operatorname{Stab}(Z)$, ne contiendrait pas d'élément d'ordre $p$, et on aurait

$$
\operatorname{deg}_{\mathscr{L}}\left(Z+\operatorname{Ker}[p]^{(v)}\right)=\frac{p^{g}}{\left|\operatorname{Ker}[p]^{(v)} \cap \operatorname{Stab}(Z)^{0}\right|} \operatorname{deg}_{\mathscr{L}}(Z),
$$

seule la composante neutre du stabilisateur intervenant au dénominateur. De plus, la variété $Z$, contenant un translaté de $V$, n'est pas incluse dans un translaté de sousvariété abélienne, ce qui entraîne :

$$
\left|\operatorname{Ker}[p]^{(v)} \cap \operatorname{Stab}(Z)^{0}\right| \leq p^{\operatorname{dim}\left(\operatorname{Stab}(Z)^{0}\right)} \leq p^{g-k^{\prime}-1} .
$$

On aurait donc

$$
\Delta^{\rho / k^{\prime}}\left(\operatorname{deg}_{\mathscr{L}}(Z)\right)^{1 / k^{\prime}}<2 \Delta^{2} \omega_{\mathscr{L}}(V) .
$$

Une contradiction suivrait immédiatement en prenant $\rho=3 k$, et en utilisant [Cha88], 2 , corollaire 2 pour extraire une hypersurface $\widetilde{Z}$ contenant $Z$ de degré

$$
\operatorname{deg}_{\mathscr{L}}(\widetilde{Z}) \leq c_{14} \operatorname{deg}_{\mathscr{L}}(Z)^{1 / k^{\prime}},
$$

pour une certaine constante $c_{14}$. On obtiendrait finalement, pour une constante $C_{\mathscr{L}}(A)$ ne dépendant que de $A$,

$$
\hat{\mu}_{\mathscr{L}}^{\mathrm{ess}}(V) \geq \frac{C_{\mathscr{L}}(A)}{\omega \mathscr{L}(V)} \log \left(3 \operatorname{deg}_{\mathscr{L}}(V)\right)^{-4 k} .
$$

5.2. Descente en codimension générale. La construction précédente ne permet pas de conclure, et on est amené à itérer, au plus $k$ fois, la dernière proposition. Cet argument de descente a déja été utilisé par Amoroso et David dans [AD99] et dans [AD03]. Il est ici compliqué par l'absence d'isogénies relevant le Frobenius en caractéristique nulle. Son absence pousse à le "reconstituer combinatoirement" en travaillant avec des réunions de translatés, et ceci explique qu' on ait écrit la partie diophantienne avec des fermés de Zariski au lieu de variétés. On va donc itérer la construction du fermé $Z$ donné par la proposition 5.1 (et en déduire l'existence d'une bonne variété), en faisant à chaque étape une hypothèse de coprimalité entre l'entier $p$ et le stabilisateur du fermé construit au cran précédent. On pourra de plus imposer un "emboîtement" entre ces variétés et on démontrera qu'il existe une suite de variétés ainsi construites associée à une suite de dimensions qui n'est pas strictement croissante. La comparaison des degrés, avec l'hypothèse de coprimalité, permettra cette fois-ci de conclure. 
Commençons par préciser les paramètres propres à la descente. Posons :

$$
\forall 1 \leq i \leq k, \quad \rho_{i}:=(5 g(k+1))^{k+1-i} ;
$$

les $\rho_{i}$ sont décroissants avec $i$. Notons aussi

$$
P_{i}:=\Delta^{\rho_{i}},
$$

où

$$
\Delta(V)=C_{0}^{2} \log \left(\left(3 \operatorname{deg}_{\mathscr{L}}(V)\right) .\right.
$$

On fixe une variété $V$ qui n'est pas incluse dans un translaté de sous-variété abélienne stricte de $A$. On va itérer la proposition 5.1 au plus $k$ fois, en employant le paramètre $\rho_{i}$ à la $i$-ème étape. Le paramètre $P_{i}$ permettra d'encadrer le premier $p_{i}$ construit. On remarque que le choix des $\rho_{i}$ et de $\Delta$ est cohérent avec les hypothèses faites au début de 5.1.

Il nous faut un nouvel indice d'obstruction pour traiter la perte de composantes dans le lemme de zéros (dont on a gardé une proportion d'au moins $\frac{1}{g+1}$ par un principe de tiroirs). Si $\Sigma$ est un sous-ensemble fini de $A$ et $V$ une sous-variété de $A$, on commence donc par noter :

$$
\alpha_{V}(\Sigma):=\text { nombre de composantes distinctes de } V+\Sigma .
$$

Définition 5.1. On définit l'indice d'obstruction de $V$ relativement à un sous-ensemble fini $\Sigma$ de $A$ :

$$
\omega_{\mathscr{L}}(V, \Sigma):=\inf _{\substack{\Lambda \subset \Sigma, \Lambda \neq \emptyset \\ V+\Lambda \subset Z}}\left\{\frac{\alpha_{V}(\Sigma)}{\alpha_{V}(\Lambda)} \operatorname{deg}_{\mathscr{L}}(Z)\right\},
$$

où $Z$ est une hypersurface (pas nécessairement irréductible).

Cette définition permet de prendre en compte la perte de translatés de $V$ en compensant cette perte par une chute du degré de la variété obstructrice correspondante.

On construit des suites de variétés ayant les propriétés suivantes :

Définition 5.2. Soit $\boldsymbol{W}(V)$ l'ensemble des quadruplets $(s, \boldsymbol{p}, \boldsymbol{\Sigma}, \boldsymbol{W})$ où $s$ est un entier tel que $1 \leq s \leq k, \boldsymbol{p}=\left(p_{1}, \ldots, p_{s}\right)$ est un $s$-uplet de nombres premiers tels que

$$
\forall 1 \leq i \leq s: \quad \frac{P_{i}}{2} \leq p_{i} \leq P_{i},
$$

l'ensemble $\boldsymbol{\Sigma}=\left(\Sigma^{1}, \ldots, \Sigma^{s}\right)$ est un $s$-uplet de sous-ensembles finis de $A$ et $\boldsymbol{W}=$ $\left(W_{0}, \ldots, W_{s}\right)$ est un $(s+1)$-uplet de sous-variétés strictes et irréductibles de $A$ avec $V=W_{0}$, et les propriétés suivantes. 
- Pour tout $1 \leq i \leq s$, l'entier $p_{i}$ est premier avec $\left[\operatorname{Stab}\left(W_{i-1}\right): \operatorname{Stab}\left(W_{i-1}\right)^{0}\right]$; il existe $v_{i} \mid p_{i}$, un premier de $\mathcal{O}_{K}$ en lequel $A$ a bonne réduction ordinaire; $\Sigma^{i}$ est un ensemble non vide tel que

$$
W_{i-1}+\Sigma^{i} \subset W_{i}
$$

- On pose $V_{0}=V$ et pour tout $1 \leq i \leq s$, on définit :

$$
V_{i}:=V+\Sigma^{1}+\cdots+\Sigma^{i} \text { et } \Lambda^{i}:=\operatorname{Ker}\left[p_{i}\right]^{\left(v_{i}\right)} .
$$

Alors on a

$$
\left(\frac{\alpha_{W_{i-1}}\left(\Lambda^{i}\right)}{\alpha_{W_{i-1}}\left(\Sigma^{i}\right)} \operatorname{deg}_{\mathscr{L}}\left(W_{i}\right)\right)^{1 / \operatorname{codim}\left(W_{i}\right)} \leq p_{i} \omega_{\mathscr{L}}\left(V_{i-1}\right)\left(\Delta^{6}\left(P_{i+1} \ldots P_{k}\right)^{g}\right) .
$$

- On a la "relation de récurrence" entre indices d'obstruction, pour tout $1 \leq i \leq s$ et $\Gamma^{i} \subset \Lambda^{i}$ :

$$
\omega_{\mathscr{L}}\left(V, \Sigma^{1}+\cdots+\Sigma^{i-1}+\Gamma^{i}\right) \leq \Delta^{3} p_{i} \omega_{\mathscr{L}}\left(V_{i-1}\right) .
$$

Remarque. Au sujet du dernier point, on convient que si $i=1$,

$$
\Sigma^{1}+\cdots+\Sigma^{i-1}+\Gamma^{i}=\Gamma^{i} .
$$

Le lemme suivant rassemble quelques propriétés immédiates des éléments de $\mathcal{W}(V)$.

Lemme 5.2. $S i(s, \boldsymbol{p}, \boldsymbol{\Sigma}, \boldsymbol{W}) \in \mathcal{W}(V)$ et $1 \leq i \leq s$, on $a$

$$
V_{i} \subset W_{i} \quad \text { et } \omega_{\mathscr{L}}\left(V_{i}\right) \leq p_{i}^{g} \omega_{\mathscr{L}}\left(V_{i-1}\right) .
$$

Preuve. L'inclusion découle de la définition 5.2 par une récurrence immédiate. Soit $Z$ une hypersurface, pas nécessairement irréductible, contenant $V_{i-1}$ et réalisant $\omega_{\mathscr{L}}\left(V_{i-1}\right)$. On a

$$
V_{i} \subset Z+\Lambda^{i}
$$

puis

$$
\omega_{\mathscr{L}}\left(V_{i}\right) \leq \operatorname{deg}_{\mathscr{L}}\left(Z+\Lambda^{i}\right) \leq\left|\Lambda^{i}\right| \operatorname{deg}_{\mathscr{L}}(Z) \leq p_{i}^{g} \omega_{\mathscr{L}}\left(V_{i-1}\right) .
$$

Notons :

$$
\mathcal{W}_{0}(V)=\left\{(s, \boldsymbol{p}, \boldsymbol{\Sigma}, \boldsymbol{W}) \in \mathcal{W}(V), \operatorname{dim}\left(W_{0}\right)<\cdots<\operatorname{dim}\left(W_{s}\right)\right\} .
$$

Si on trouve un quadruplet $(s, \boldsymbol{p}, \boldsymbol{\Sigma}, \boldsymbol{W}) \in \mathcal{W} \backslash \mathcal{W}_{0}$, la comparaison des degrés aux variétés de même dimension permet de trouver une contradiction : 
Proposition 5.3. On a l'égalité $W_{0}(V)=\mathcal{W}(V)$.

Preuve. Supposons par l'absurde qu'il existe $(s, \boldsymbol{p}, \boldsymbol{\Sigma}, \boldsymbol{W}) \in \mathcal{W}(V) \backslash \mathcal{W}_{0}(V)$. On peut donc trouver $1 \leq i \leq s$ tel que :

$$
\operatorname{dim}\left(W_{i-1}\right)=\operatorname{dim}\left(W_{i}\right) \quad \text { et } \quad W_{i-1}+\Sigma^{i} \subset W_{i} .
$$

On observe d'abord que pour tout $0 \leq j \leq s, W_{j}$ contient un translaté de $V$. Comme $V$ n'est inclus dans aucun translaté d'une sous-variété abélienne stricte, il en va donc de même pour $W_{j}$.

Par irréductibilité de $W_{i}$, on observe que $\alpha_{W_{i-1}}\left(\Sigma^{i}\right)=1$. Comme $p_{i}$ est premier avec $\left[\operatorname{Stab}\left(W_{i-1}\right): \operatorname{Stab}\left(W_{i-1}\right)^{0}\right]$, les éléments du groupe $\operatorname{Stab}\left(W_{i-1}\right) / \operatorname{Stab}\left(W_{i-1}\right)^{0}$, qui se relève dans $\operatorname{Stab}\left(W_{i-1}\right)$, ne sont pas d'ordre $p_{i}$. On a donc

$$
\begin{aligned}
\alpha_{W_{i-1}}\left(\Lambda^{i}\right) & =\frac{\left|\Lambda^{i}\right|}{\left|\Lambda^{i} \cap \operatorname{Stab}\left(W_{i-1}\right)\right|} \\
& =\frac{\left|\operatorname{Ker}\left[p_{i}\right]^{\left({ }_{i}\right)}\right|}{\left|\operatorname{Ker}\left[p_{i}\right]^{\left(v_{i}\right)} \cap \operatorname{Stab}\left(W_{i-1}\right)^{0}\right|} \\
& \geq \frac{p_{i}^{g}}{p_{i}^{\operatorname{dim}\left(\operatorname{Stab}\left(W_{i-1}\right)^{0}\right)} \geq p_{i}^{1+\operatorname{codim}\left(W_{i}\right)},}
\end{aligned}
$$

la dernière inégalité provenant du fait que $W_{i-1}$ n'est pas un translaté de sous-variété abélienne. On en déduit :

$$
p_{i}\left(p_{i} \operatorname{deg}_{\mathscr{L}}\left(W_{i-1}\right)\right)^{1 / \operatorname{codim}\left(W_{i}\right)} \leq p_{i} \omega_{\mathscr{L}}\left(V_{i-1}\right) \Delta^{6}\left(P_{i+1} \ldots P_{k}\right)^{g} .
$$

Mais par choix des paramètres,

$$
p_{i}^{-1 / \operatorname{codim}\left(W_{i}\right)} \Delta^{6}\left(P_{i+1} \ldots P_{k}\right)^{g} \leq 2 \Delta^{u},
$$

pour $u \in \mathbb{R}$. En remarquant que les $\rho_{i}$ sont une suite géométrique et que $g \geq 2$, on a :

$$
\begin{aligned}
k u & =-\rho_{i} k / \operatorname{codim}\left(W_{i-1}\right)+6 k+k g\left(\rho_{i+1}+\cdots+\rho_{k}\right) \\
& \leq-\rho_{i}+5 k g \rho_{i+1} \\
& \leq-5(k+1) g \rho_{i+1}+5 k g \rho_{i+1}<0 .
\end{aligned}
$$

Par le lemme 5.2, on a $V_{i-1} \subset W_{i-1}$, ce qui entraîne, par le théorème de Chardin et le choix de $\Delta$ :

$$
\omega_{\mathscr{L}}\left(V_{i-1}\right) \leq \Delta^{u / 2} \omega_{\mathscr{L}}\left(V_{i-1}\right) .
$$

Puisque l'indice d'obstruction est non nul, on en déduit une contradiction. 
5.3. Un dernier argument géométrique. Dans le paragraphe précédent, on avait seulement besoin de savoir que $V$ n'est pas incluse dans un translaté de sous-variété abélienne. On suppose désormais :

$$
\hat{\mu}_{\mathscr{L}}^{\mathrm{ess}}(V) \omega_{\mathscr{L}}(V)<\frac{1}{\Delta^{(5 g(k+1))^{k+1}}} .
$$

La preuve du théorème 1.1 sera achevée si on réussit à démontrer la négation de la proposition 5.3, à savoir :

Proposition 5.4. On a $\mathfrak{W}_{0}(V) \neq \mathcal{W}(V)$.

Commençons par quelques préliminaires. À un élément $(s, \boldsymbol{p}, \boldsymbol{\Sigma}, \boldsymbol{W})$ de $\mathcal{W}$, on peut associer la suite de longueur $s+1$, croissante, des dimensions des $W_{i}$. On commence par construire un ordre total sur ces suites. Soient $v=\left(v_{i}\right)_{0 \leq i \leq s}$ et $v^{\prime}=\left(v_{i}^{\prime}\right)_{0 \leq i \leq s^{\prime}}$ deux suites croissantes d'entiers positifs.

Définition 5.3. On dit que $v \preceq v^{\prime} \operatorname{si}\left(v_{i}\right)_{0 \leq i \leq \min \left\{s, s^{\prime}\right\}}<\left(v_{i}^{\prime}\right)_{0 \leq i \leq \min \left\{s, s^{\prime}\right\}}$ pour l'ordre lexicographique ou, en cas d'égalité, si $s \geq s^{\prime}$.

Remarque. Si une suite est la tronquée d'une autre suite, elle est plus grande selon cet ordre (il y a un renversement des inégalités).

La proposition 5.4 se démontre par un argument de descente : on prend un élément $(s, \boldsymbol{p}, \boldsymbol{\Sigma}, \boldsymbol{W}) \in \mathcal{W}_{0}(V)$ associé à une suite minimale, et on construit un nouvel élément $\left(s^{\prime}, \boldsymbol{p}^{\prime}, \boldsymbol{\Sigma}^{\prime}, \boldsymbol{W}^{\prime}\right)$ dans $\mathcal{W}(V)$, associé à une suite strictement inférieure; cet élément est alors nécessairement dans $\mathcal{W}(V) \backslash \mathcal{W}_{0}(V)$.

Comme il n'y a qu'un nombre fini de suites de $k+1$ entiers entre 0 et $k$, les suites croissantes de dimensions associées aux éléments de $\mathcal{W}_{0}(V)$ sont en nombre fini.

Lemme 5.5. On $a \mathcal{W}_{0}(V) \neq \varnothing$.

Preuve. Compte tenu de la proposition 5.3, on peut se contenter de trouver un élément dans $\mathcal{W}(V)$. On remarque d'abord, par le lemme 2.1 (ii) de [DH00] :

$$
\log \left(\left[\operatorname{Stab}(V): \operatorname{Stab}(V)^{0}\right]\right) \leq \log \left(\operatorname{deg}_{\mathscr{L}}(\operatorname{Stab}(V))\right) \leq g \log \left(\operatorname{deg}_{\mathscr{L}}(V)\right) \leq \Delta .
$$

La procédure diophantienne (proposition 5.1) avec $X=V$ et $R=[\operatorname{Stab}(V)$ : $\operatorname{Stab}(V)^{0}$ ] donne l'existence d'un entier $p_{1}$ tel que :

$$
\frac{P_{1}}{2} \leq p_{1} \leq P_{1}
$$

et d'un fermé $W_{1}$ contenant $W_{0}+\Lambda^{1}$ tel que :

$$
\operatorname{deg}_{\mathscr{L}}\left(W_{1}\right)^{1 / \operatorname{codim}\left(W_{1}\right)} \leq p_{1} \Delta^{2} \omega_{\mathscr{L}}(V) .
$$


De plus, le premier $p_{1}$ est premier à $\left[\operatorname{Stab}\left(W_{0}\right): \operatorname{Stab}\left(W_{0}\right)^{0}\right]$ par construction.

La relation de récurrence entre indices d'obstruction, pour tout $\Gamma^{1} \subset \Lambda^{1}$, vient en extrayant une hypersurface $Z_{1}$ contenant $W_{1}$ par le théorème de Chardin ([Cha88], 2, corollaire 2) :

$$
\omega \mathscr{L}\left(V, \Gamma^{1}\right) \leq \operatorname{deg}_{\mathscr{L}}\left(Z_{1}\right) \leq p_{1} \Delta^{3} \omega \mathscr{L}(V) .
$$

La constante de comparaison entre les degrés dans le théorème de Chardin est éliminée en remplaçant $\Delta^{2}$ par $\Delta^{3}$.

On peut donc choisir une suite minimale pour l'ordre $\preceq$, associée à un quadruplet $(s, \boldsymbol{p}, \boldsymbol{\Sigma}, \boldsymbol{W})$. Le lemme technique suivant montre comment construire l'élément $\left(s^{\prime}, \boldsymbol{p}^{\prime}, \boldsymbol{\Sigma}^{\prime}, \boldsymbol{W}^{\prime}\right)$ en utilisant le théorème de Bézout. Il restera ensuite à prouver que ce quadruplet est encore dans $\mathcal{W}(V)$.

Lemme 5.6. Il existe un entier $\frac{P_{s+1}}{2} \leq p_{s+1} \leq P_{s+1}$ premier avec $\left[\operatorname{Stab}\left(W_{s}\right)\right.$ : $\left.\operatorname{Stab}\left(W_{s}\right)^{0}\right]$, un indice $1 \leq s^{\prime} \leq s+1$, un sous-ensemble $\widetilde{\Sigma}^{s^{\prime}}$ de $\Lambda^{s^{\prime}}$ et une sousvariété $Z_{s^{\prime}}$ stricte et irréductible, dont le degré vérifie

$\frac{\alpha_{W_{s^{\prime}-1}}\left(\Lambda^{s^{\prime}}\right)}{\alpha_{W_{s^{\prime}-1}}\left(\widetilde{\Sigma^{s^{\prime}}}\right)} \operatorname{deg}_{\mathscr{L}}\left(Z_{s^{\prime}}\right) \leq \frac{\alpha_{W_{s^{\prime}-1}}\left(\Lambda^{s^{\prime}}\right)}{\alpha_{W_{s^{\prime}-1}}\left(\Sigma^{s^{\prime}}\right)} \operatorname{deg}_{\mathscr{L}}\left(W_{s^{\prime}}\right) p_{s^{\prime}} \omega_{\mathscr{L}}\left(V_{s^{\prime}-1}\right)\left(\Delta^{6} p_{s^{\prime}+1}^{g} \ldots p_{s+1}^{g}\right)$,

et telle qu'on ait l'inclusion

$$
W_{s^{\prime}-1}+\widetilde{\Sigma}^{s^{\prime}} \subset Z_{s^{\prime}}
$$

avec

$$
\operatorname{codim}\left(Z_{s^{\prime}}\right)=\operatorname{codim}\left(W_{s^{\prime}}\right)+1,
$$

en posant les conventions $: \operatorname{codim}\left(W_{s+1}\right)=0, \operatorname{deg}_{\mathscr{L}}\left(W_{s+1}\right)=1$ et $\Sigma^{s+1}=\Lambda^{s+1}$.

Remarque. Avec la définition de l'ordre $\prec$, on vient bien de construire une suite de dimensions "plus petite" :

$$
\left(\operatorname{dim}\left(W_{0}\right), \ldots, \operatorname{dim}\left(W_{s^{\prime}-1}\right), \operatorname{dim}\left(Z_{s^{\prime}}\right)\right) \prec\left(\operatorname{dim}\left(W_{0}\right), \ldots, \operatorname{dim}\left(W_{s}\right)\right) .
$$

Preuve. On va utiliser la proposition 5.1 avec

$$
\rho=\rho_{s+1} \quad \text { et } \quad R=\left[\operatorname{Stab}\left(W_{s}\right): \operatorname{Stab}\left(W_{s}\right)^{0}\right] \prod_{i=1}^{s} p_{i}
$$

On pose

$$
\Sigma_{0}=\Sigma^{1}+\cdots+\Sigma^{s},
$$


puis on prend $\Sigma$ tel qu'il existe $Z$ contenant $V+\Sigma$ et vérifiant

$$
\omega_{\mathscr{L}}\left(V, \Sigma_{0}\right)=\frac{\alpha_{V}\left(\Sigma_{0}\right)}{\alpha_{V}(\Sigma)} \operatorname{deg}_{\mathscr{L}}(Z) .
$$

On choisit alors

$$
X=V+\Sigma \text {. }
$$

Le fermé $X$ étant réunion d'un nombre fini de translatés de $V$ par des points de hauteur nulle, on a

$$
\hat{\mu}_{\mathscr{L}}^{\mathrm{ess}}(X)=\hat{\mu}_{\mathscr{L}}^{\mathrm{ess}}(V) .
$$

De plus, le lemme 5.2 itéré donne

$$
\omega_{\mathscr{L}}(X) \leq \omega_{\mathscr{L}}\left(V_{s}\right) \leq p_{1}^{g} \ldots p_{s}^{g} \omega_{\mathscr{L}}(V) \leq P_{1}^{g} \ldots P_{s}^{g} \omega_{\mathscr{L}}(V)
$$

Il en résulte

$$
\begin{aligned}
\omega_{\mathscr{L}}(X) \hat{\mu}_{\mathscr{L}}^{\mathrm{ess}}(X) & \leq P_{1}^{g} \ldots P_{s}^{g} \omega_{\mathscr{L}}(V) \hat{\mu}_{\mathscr{L}}^{\mathrm{ess}}(V) \\
& \leq \Delta^{u}
\end{aligned}
$$

où

$$
\begin{aligned}
u & =-(5 g(k+1))^{k+1}+g\left(\rho_{1}+\cdots+\rho_{s}\right) \\
& \leq-(5 g(k+1))^{k+1}+g\left(\rho_{1}+\cdots+\rho_{s+1}\right)-\rho_{s+1} \\
& \leq \rho_{1}(2 g-5 g(k+1))-\rho_{s+1} \\
& <-1-\rho_{s+1} .
\end{aligned}
$$

L'hypothèse de la proposition 5.1 est donc vérifiée avec $\rho=\rho_{s+1}$. De plus :

$$
C_{0} \log (3 \omega \mathscr{L}(X)) \leq C_{0}\left(g \log P_{1}+\cdots+g \log P_{S}+\log (3 \omega \mathscr{L}(V))\right) \leq \Delta(V) .
$$

On majore ensuite $\log (R)$, en remarquant que

$$
\log (R) \leq \log \operatorname{deg}\left(\operatorname{Stab}\left(W_{s}\right)\right)+\log \left(P_{1}\right)+\cdots+\log \left(P_{S}\right),
$$

puis de la même façon que dans la preuve du lemme 5.5 :

$$
\begin{aligned}
\log (R) & \leq\left(\operatorname{dim}\left(W_{s}\right)+1\right) \log \left(\operatorname{deg}_{\mathscr{L}}\left(W_{s}\right)\right)+\frac{1}{2} \Delta(V) \\
& \leq g\left(g\left(\log \Delta+\log P_{s}+\cdots+\log P_{k}\right)+g \log \left(\omega_{\mathscr{L}}\left(V_{s-1}\right)\right)\right)+\frac{1}{2} \Delta(V) \\
& \leq \Delta(V) .
\end{aligned}
$$


La proposition 5.1 donne donc l'existence d'un entier

$$
\frac{P_{s+1}}{2} \leq p_{s+1} \leq P_{s+1},
$$

strictement positif, d'un fermé de Zariski équidimensionnel $Z_{0}$, de codimension $k^{\prime}$, tel que le nombre de composantes distinctes de $X$ dans $Z_{0}$ soit supérieur ou égal à $\frac{\alpha_{V}(\Sigma)}{g}$, et vérifiant l'inégalité suivante :

$$
\operatorname{deg}_{\mathscr{L}}\left(Z_{0}+\Lambda^{s+1}\right)^{1 / k^{\prime}}<\Delta^{2} p_{s+1} \omega_{\mathscr{L}}(X) .
$$

De plus, le théorème d'interpolation de Chardin permet d'extraire une hypersurface $\widetilde{Z}$, équidimensionnelle mais pas nécessairement irréductible, contenant $Z_{0}+\Lambda^{s+1}$ telle que, pour une constante $c_{15}$ ne dépendant que de $A$,

$$
\operatorname{deg}_{\mathscr{L}}(\widetilde{Z}) \leq c_{15} \operatorname{deg}_{\mathscr{L}}\left(Z_{0}+\Lambda^{s+1}\right)^{1 / k^{\prime}} \leq c_{15} \Delta^{2} p_{s+1} \omega_{\mathscr{L}}(X) .
$$

On peut maintenant démontrer l'inégalité entre les indices d'obstruction au "cran $s+1$ ”. Remarquons que si $\Gamma^{s+1}$ est un sous-ensemble quelconque de $\Lambda^{s+1}$, on a

$$
\begin{aligned}
\omega_{\mathscr{L}}\left(V, \Sigma_{0}+\Gamma^{s+1}\right) & \leq g \frac{\alpha_{V}\left(\Sigma_{0}+\Gamma^{s+1}\right)}{\alpha_{V}\left(\Sigma+\Gamma^{s+1}\right)} \operatorname{deg}_{\mathscr{L}}(\tilde{Z}) \\
& \leq \Delta^{3} p_{s+1} \frac{\alpha_{V}\left(\Sigma_{0}\right) \alpha_{V}\left(\Gamma^{s+1}\right)}{\alpha_{V}(\Sigma) \alpha_{V}\left(\Gamma^{s+1}\right)} \omega_{\mathscr{L}}(V+\Sigma) .
\end{aligned}
$$

On a fait apparaître les facteurs $\alpha_{V}\left(\Gamma^{s+1}\right)$ en remarquant que les points de $\Gamma^{s+1}$ sont d'ordre premier aux ordres des points de $\Sigma^{i}$, pour tout $1 \leq i \leq s$. On utilise alors les propriétés fines des nouveaux indices d'obstruction, et la définition de $\Sigma$, qui garantit l'existence d'une hypersurface $Z$ contenant $V+\Sigma$ telle que

$$
\omega_{\mathscr{L}}\left(V+\Sigma_{0}\right) \geq \omega_{\mathscr{L}}\left(V, \Sigma_{0}\right)=\frac{\alpha_{V}\left(\Sigma_{0}\right)}{\alpha_{V}(\Sigma)} \operatorname{deg}_{\mathscr{L}}(Z) \geq \frac{\alpha_{V}\left(\Sigma_{0}\right)}{\alpha_{V}(\Sigma)} \omega_{\mathscr{L}}(V+\Sigma) .
$$

On en déduit l'inégalité suivante :

$$
\omega_{\mathscr{L}}\left(V, \Sigma_{0}+\Gamma^{s+1}\right) \leq \Delta^{3} p_{s+1} \omega_{\mathscr{L}}\left(V+\Sigma_{0}\right) .
$$

Premier cas. L'hypersurface $\widetilde{Z}$ contient $W_{s}+\Lambda^{s+1}$. On prend une composante irréductible $Z_{s+1}$ de $\widetilde{Z}$ "au-dessus de la moyenne" dans le sens suivant. Il existe $\widetilde{\Sigma}^{s+1}$ un sous-ensemble fini et non-vide de $\Lambda^{s+1}$ tel qu' on ait

$$
W_{s}+\tilde{\Sigma}^{s+1} \subset Z_{s+1},
$$

et que

$$
\frac{\operatorname{deg}_{\mathscr{L}}\left(Z_{s+1}\right)}{\operatorname{deg}_{\mathscr{L}}(\widetilde{Z})} \leq \frac{\alpha_{W_{s}}\left(\widetilde{\Sigma}^{s+1}\right)}{\alpha_{W_{s}}\left(\Sigma^{s+1}\right)}
$$


Si on note $\tilde{Z}=\bigcup_{\beta} Z_{\beta}$ la décomposition de $\tilde{Z}$ en composantes irréductibles et si $\Sigma^{(\beta)}$ est l'ensemble des $x \in \Sigma^{s+1}$ tels que $x+W_{s} \subset Z_{\beta}$, on a en effet

$$
\frac{\sum_{\beta} \operatorname{deg}_{\mathscr{L}}\left(Z_{\beta}\right)}{\operatorname{deg}_{\mathscr{L}}(\tilde{Z})}=\frac{\sum_{\beta} \alpha_{W_{s}}\left(\Sigma^{(\beta)}\right)}{\alpha_{W_{S}}\left(\Sigma^{s+1}\right)}=1,
$$

et l'existence de $Z_{s+1}$ vérifiant (4) suit. L'inégalité du lemme est alors démontrée avec $s^{\prime}=s+1$, en remarquant que $\Lambda^{s+1}=\Sigma^{s+1}$ :

$$
\frac{\alpha_{W_{S}}\left(\Sigma^{s+1}\right)}{\alpha_{W_{S}}\left(\widetilde{\Sigma}^{s+1}\right)} \operatorname{deg}_{\mathscr{L}}\left(Z_{s+1}\right) \leq \Delta^{3} p_{s+1} \omega \mathscr{L}(X) \leq \Delta^{3} p_{s+1} \omega \mathscr{L}\left(V_{s}\right) .
$$

Deuxième cas. Le fermé $\widetilde{Z}$ ne contient pas

$$
W_{s}+\Lambda^{s+1}=W_{s}+\Sigma^{s+1} .
$$

Remarquons que translater $\tilde{Z}$ ne change rien à son degré. Soit $s^{\prime}$ le plus grand entier $\leq s$ tel qu'il existe un ensemble $\Gamma^{s^{\prime}}$ avec (quitte à translater $\widetilde{Z}$ par un point de torsion)

$$
W_{s^{\prime}-1}+\Gamma^{s^{\prime}} \subset \tilde{Z},
$$

vérifiant que

$$
\frac{\alpha_{W_{s^{\prime}-1}}\left(\Gamma^{s^{\prime}}\right)}{\alpha_{W_{s^{\prime}-1}}\left(\Sigma^{s^{\prime}}\right)} \geq \frac{1}{2 g} \frac{\alpha_{V}(\Sigma)}{\alpha_{V}\left(\Sigma_{0}\right)} ;
$$

et tel que cette propriété soit vérifiée pour tout entier $\leq s^{\prime}$. Comme $V=W_{0}$, la construction de $\widetilde{Z}$ garantit que $s^{\prime} \geq 1$ (la perte de composantes au premier cran ne peut être plus grande que la perte totale).

Cette clause technique tient au fait que la variété obstructrice dans le lemme de zéros ne contient pas nécessairement toutes les composantes de $V+\Sigma_{0}$, mais une proportion importante par un principe de tiroirs. On a donc introduit le sous-ensemble $\Sigma$ de $\Sigma_{0}$ qu'on ne contrôle pas et (5) montre que la perte de composantes dans la descente est limitée. Elle sera compensée par les propriétés des indices d'obstruction en jeu.

Au cran suivant, on observe en particulier que

$$
W_{s^{\prime}}+\Sigma^{s^{\prime}+1} \nsubseteq \tilde{Z},
$$

et on peut supposer sans perte de généralité que

$$
W_{s^{\prime}} \not \subset \tilde{Z} \text {. }
$$

Par construction, il existe un sous-ensemble $\Gamma^{s^{\prime}}$ de $\Sigma^{s^{\prime}}$ vérifiant (5). Les deux fermés $W_{s^{\prime}}$ et $\widetilde{Z}$ contiennent alors $W_{s^{\prime}-1}+\Gamma^{s^{\prime}}$ (voir la définition 5.2). Comme $W_{s^{\prime}}$ est 
irréductible et n'est incluse dans aucune des composantes de $\widetilde{Z}$, qui sont toutes de codimension 1, l'intersection $W_{s^{\prime}} \cap \widetilde{Z}$ est un fermé équidimensionnel de dimension $\operatorname{dim}\left(W_{s^{\prime}}\right)-1$ et contient encore le fermé $W_{s^{\prime}-1}+\Gamma^{s^{\prime}}$. De plus, son degré est majoré par le théorème de Bézout (quitte à sommer les degrés sur toutes les composantes irréductibles de $\widetilde{Z}$ ) :

$$
\operatorname{deg}_{\mathscr{L}}\left(W_{s^{\prime}} \cap \widetilde{Z}\right) \leq \operatorname{deg}_{\mathscr{L}}\left(W_{s^{\prime}}\right) \operatorname{deg}_{\mathscr{L}}(\widetilde{Z}) .
$$

On choisit alors, comme dans le premier cas, une composante irréductible $Z_{s^{\prime}}$ de cette intersection "meilleure que la moyenne", c'est-à-dire telle qu'il existe $\widetilde{\Sigma}^{s^{\prime}}$ avec

$$
W_{s^{\prime}-1}+\widetilde{\Sigma}^{s^{\prime}} \subset Z_{s^{\prime}}
$$

et qu'on ait l'inégalité suivante :

$$
\frac{\operatorname{deg}_{\mathscr{L}}\left(Z_{s^{\prime}}\right)}{\operatorname{deg}_{\mathscr{L}}\left(W_{s^{\prime}} \cap \tilde{Z}\right)} \leq \frac{\alpha_{W_{s^{\prime}-1}}\left(\widetilde{\Sigma}^{s^{\prime}}\right)}{\alpha_{W_{s^{\prime}-1}}\left(\Gamma^{s^{\prime}}\right)}
$$

On en déduit :

$$
\frac{\alpha_{W_{s^{\prime}-1}}\left(\Lambda^{s^{\prime}}\right)}{\alpha_{W_{s^{\prime}-1}}\left(\widetilde{\Sigma}^{s^{\prime}}\right)} \operatorname{deg}_{\mathscr{L}}\left(Z_{s^{\prime}}\right) \leq \frac{\alpha_{W_{s^{\prime}-1}}\left(\Lambda^{s^{\prime}}\right)}{\alpha_{W_{s^{\prime}-1}}\left(\Sigma^{s^{\prime}}\right)} \operatorname{deg}_{\mathscr{L}}\left(W_{s^{\prime}}\right)\left(\Delta^{3} p_{s+1} \frac{\alpha_{V}\left(\Sigma_{0}\right)}{\alpha_{V}(\Sigma)} \omega_{\mathscr{L}}(X)\right) .
$$

A ce stade, on utilise les remarques précédant (3), en rappelant que $X=V+\Sigma$ :

$$
\frac{\alpha_{V}\left(\Sigma_{0}\right)}{\alpha_{V}(\Sigma)} \omega_{\mathscr{L}}(X) \leq \omega_{\mathscr{L}}\left(V, \Sigma_{0}\right)
$$

De plus, soit $Z$ contenant un certain $V+\Lambda$ et réalisant $\omega_{\mathscr{L}}\left(V, \Sigma^{1}+\cdots+\Sigma^{s^{\prime}}\right)$. On a alors, quitte à considérer un translaté de $Z$ et en faisant une majoration grossière :

$$
\omega_{\mathscr{L}}\left(V, \Sigma_{0}\right) \leq \frac{\alpha_{V}\left(\Sigma_{0}\right)}{\alpha_{V}(\Lambda)} \operatorname{deg}_{\mathscr{L}}(Z) \leq p_{s^{\prime}+1}^{g} \ldots p_{s}^{g} \omega_{\mathscr{L}}\left(V, \Sigma^{1}+\cdots+\Sigma^{s^{\prime}}\right) .
$$

Il reste enfin à utiliser la relation de récurrence de la définition 5.2 :

$$
\omega_{\mathscr{L}}\left(V, \Sigma^{1}+\cdots+\Sigma^{s^{\prime}}\right) \leq \Delta^{3} p_{s^{\prime}} \omega_{\mathscr{L}}\left(V_{s^{\prime}-1}\right) .
$$

En mettant bout à bout ces inégalités, il vient

$$
\begin{aligned}
& \frac{\alpha_{W_{s^{\prime}-1}}\left(\Lambda^{s^{\prime}}\right)}{\alpha_{W_{s^{\prime}-1}}\left(\widetilde{\Sigma}^{s^{\prime}}\right)} \operatorname{deg}_{\mathscr{L}}\left(Z_{s^{\prime}}\right) \\
& \quad \leq \frac{\alpha_{W_{s^{\prime}-1}}\left(\Lambda^{s^{\prime}}\right)}{\alpha_{W_{s^{\prime}-1}}\left(\Sigma^{s^{\prime}}\right)} \operatorname{deg}_{\mathscr{L}}\left(W_{s^{\prime}}\right) p_{s^{\prime}} \omega \mathscr{L}\left(V_{s^{\prime}-1}\right)\left(\Delta^{6} p_{s^{\prime}+1}^{g} \ldots p_{s+1}^{g}\right) .
\end{aligned}
$$


La proposition 5.4 sera prouvée si on parvient à démontrer que la nouvelle suite $\left(s^{\prime}, \boldsymbol{l}^{\prime}, \boldsymbol{\Sigma}^{\prime}, \boldsymbol{W}^{\prime}\right)$ construite dans le lemme précédent est dans $\mathcal{W}(V)$ :

Lemme 5.7. La suite $\left(s^{\prime}, \boldsymbol{l}^{\prime}, \boldsymbol{\Sigma}^{\prime}, \boldsymbol{W}^{\prime}\right)$ est dans $\mathcal{W}(V)$.

Preuve. Rappelons que la nouvelle suite est définie par

$$
\Sigma^{\prime}=\left(\Sigma^{1}, \ldots, \Sigma^{s^{\prime}-1}, \widetilde{\Sigma}^{s^{\prime}}\right)
$$

et

$$
\boldsymbol{W}^{\prime}=\left(W_{0}, \ldots, W_{s^{\prime}-1}, Z_{s^{\prime}}\right) .
$$

Le premier point est toujours vérifié par construction.

Démontrons le second point pour $i=s^{\prime}$, les autres cas étant inchangés. Si $s^{\prime} \neq$ $s+1$, on a

$$
\begin{aligned}
& \frac{\alpha_{W_{s^{\prime}-1}}\left(\Lambda^{s^{\prime}}\right)}{\alpha_{W_{s^{\prime}-1}}\left(\widetilde{\Sigma}^{s^{\prime}}\right)} \operatorname{deg}_{\mathscr{L}}\left(Z_{s^{\prime}}\right) \\
& \quad \leq \frac{\alpha_{W_{s^{\prime}-1}}\left(\Lambda^{s^{\prime}}\right)}{\alpha_{W_{s^{\prime}-1}}\left(\Sigma^{s^{\prime}}\right)} \operatorname{deg}_{\mathscr{L}}\left(W_{s^{\prime}}\right) p_{s^{\prime}} \omega_{\mathscr{L}}\left(V_{s^{\prime}-1}\right) \Delta^{6}\left(p_{s^{\prime}+1} \ldots p_{s+1}\right)^{g} \\
& \leq\left(p_{s^{\prime}} \omega_{\mathscr{L}}\left(V_{s^{\prime}-1}\right) \Delta^{6}\left(P_{s^{\prime}+1} \ldots P_{k}\right)^{g}\right)^{\operatorname{codim}\left(W_{s^{\prime}}\right)} p_{s^{\prime}} \omega_{\mathscr{L}}\left(V_{s^{\prime}-1}\right) \Delta^{6}\left(p_{s^{\prime}+1} \ldots p_{s+1}\right)^{g} \\
& \leq\left(p_{s^{\prime}} \omega_{\mathscr{L}}\left(l_{s^{\prime}}, V_{s^{\prime}-1}\right) \Delta^{6}\left(P_{s^{\prime}+1} \ldots P_{k}\right)^{g}\right)^{\operatorname{codim}\left(Z_{s^{\prime}}\right)},
\end{aligned}
$$

puisque dans ce cas $: \operatorname{codim}\left(Z_{s^{\prime}}\right)=\operatorname{codim}\left(W_{s^{\prime}}\right)+1$. Si $s^{\prime}=s+1$, la majoration du lemme 5.6 donne directement le résultat.

Enfin, la "relation de récurrence" entre indices d'obstruction est trivialement réalisée pour $i \neq s^{\prime}$; pour $i=s^{\prime}=s+1$, c'est l'inégalité (3); et pour $i=s^{\prime}$ et $s^{\prime} \neq s+1$, il n'y a rien à montrer.

Preuve du théorème 1.1. Soit $V$ une sous-variété stricte de $A$ qui n'est pas incluse dans un translaté de sous-variété abélienne stricte de $A$. Par les propositions 5.3 et 5.4, la variété $V$ contredit l'inégalité suivante :

$$
\hat{\mu}_{\mathscr{L}}^{\mathrm{ess}}(V) \omega \mathscr{L}(V)<\frac{1}{\Delta^{(5 g(k+1))^{k+1}}},
$$

avec $\Delta=C_{0}^{2} \log \left(3 \operatorname{deg}_{\mathscr{L}}(V)\right)$. On en déduit :

$$
\hat{\mu}_{\mathscr{L}}^{\mathrm{ess}}(V) \geq \frac{C_{\mathscr{L}}(A)}{\omega_{\mathscr{L}}(V)} \times\left(\log \left(3 \operatorname{deg}_{\mathscr{L}}(V)\right)\right)^{-\lambda(k)},
$$

où $\lambda(k)=(5 g(k+1))^{k+1}$ et $C_{\mathscr{L}}(A)=\frac{1}{C_{0}^{2 \lambda(g)}}$, qui ne dépend que de $A$. 


\section{Références}

[Amo07] F. Amoroso, Bogomolov on tori revisited. http ://hal.archives-ouvertes.fr/hal-00132119/fr/

[AD99] F. Amoroso et S. David, Le problème de Lehmer en dimension supérieure. J. Reine Angew. Math. 513 (1999), 145-179. Zbl 1011.11045 MR 1713323

[AD03] F. Amoroso et S. David, Minoration de la hauteur normalisée dans un tore. J. Inst. Math. Jussieu 2 (3) (2003), 335-381. Zbl 1041.11048 MR 1990219

[BS04] M. Baker et J. Silverman, A lower bound for the canonical height on abelian varieties over abelian extensions. Math. Res. Lett. 11 (2004), 377-396. Zbl 1060.11041 MR 2067482

[BMZ99] E. Bombieri, D. Masser et U. Zannier, Intersecting a curve with algebraic subgroups of multiplicative groups. Internat. Math. Res. Notices 20 (1999), 1119-1140. Zbl 0938.11031 MR 1728021

[BZ95] E. Bombieri et U. Zannier, Algebraic points on subvarieties of $\mathbb{G}_{m}^{n}$. Internat. Math. Res. Notices 7 (1995), 333-347. Zbl 0848.11030 MR 1350686

[BZ96] E. Bombieri et U. Zannier, Heights of algebraic points on subvarieties of abelian varieties. Ann. Scuola Norm. Sup. Pisa Cl. Sci. (4) 23 (1996), no. 4, 779-792. Zbl 0897.11020 MR 1469574

[BGS94] J. B. Bost, H. Gillet et C. Soulé, Heights of projective varieties. J. Amer. Math. Soc. 7 (1994), no. 4, 903-1027, Zbl 0973.14013 MR 1260106

[Car08] M. Carrizosa, Problème de Lehmer et variétés abéliennes CM. C. R. Math. Acad. Sci. Paris 346 (2008), no. 23-24, 1219-1224. Zbl 05486440 MR 2473296

[Cha88] M. Chardin, Une majoration de la fonction de Hilbert et ses conséquences pour l'interpolation algébrique. Bull. Soc. Math. France 117 (1988), 305-318. Zbl 0709.13007 MR 1020108

[Dav89] S. David, Fonctions thêta, formes modulaires et approximation diophantienne. Thèse de Doctorat, Université Paris VI, 1989.

[Dav91] S. David, Fonctions thêta et points de torsion des variétés abéliennes. Compositio Math. 78 (1991), no. 2, 121-160, 1991. Zbl 0741.14025 MR 1104784

[DH00] S. David et M. Hindry, Minoration de la hauteur de Néron-Tate sur les variétés abéliennes de type C.M. J. Reine Angew. Math. 529 (2000), 1-74. Zbl 0993.11034 MR 1799933

[DP00] S. David et P. Philippon, Sous-variétés de torsion des variétés semi-abéliennes. C. R. Acad. Sci. Paris Sér. I Math. 331 (2000), no. 8, 587-592. Zbl 0972.11059 MR 1799094

[DP02] S. David et P. Philippon, Minoration des hauteurs normalisées des sous-variétés de variétés abéliennes II. Comment. Math. Helv. 77 (2002), 639-700. Zbl 1030.11026 MR 1949109

[Dob79] E. Dobrowolski, On a question of Lehmer and the number of irreducible factors of a polynomial. Acta Arith. 34 (1979), 391-401. Zbl 0416.12001 MR 0543210

[Gal08] A. Galateau, Le problème de Bogomolov effectif sur les variétés abéliennes. Prépublication 2008, arXiv:0805.2663v1 [math.NT]. 
Vol. 85 (2010) Une minoration du minimum essentiel sur les variétés abéliennes

[Hab08] P. Habegger, A Bogomolov property for curves modulo algebraic subgroups. Bull. Soc. Math. France 137 (2009), no. 1, 93-125. Zbl 05558321 MR 2496702

[Har77] R. Hartshorne, Algebraic Geometry. Grad. Texts in Math. 52, Springer-Verlag, New York 1977. Zbl 0367.14001 MR 0463157

[HS00] M. Hindry et J. Silverman, Diophantine Geometry : An Introduction. Grad. Texts in Math. 201, Springer-Verlag, New York 2000. Zbl 0948.11023 MR 1745599

[LR85] H. Lange et W. Ruppert, Complete systems of addition laws on abelian varieties. Invent. Math. 79 (1985), no. 3, 603-610. Zbl 0577.14035 MR 0782238

[Lau83] M. Laurent, Minoration de la hauteur de Néron-Tate. In Séminaire de théorie des nombres, Paris, 1981-1982, Progr. Math. 38, Boston, Mass., 1983, 137-152. Zbl 0521.14010 MR 0729165

[Lit99] R. Litcanu, Minoration des hauteurs des sous-variétés de variétés abéliennes. Etude du degré des morphismes de Belyi. Thèse de Doctorat, Université Paris XI, 1999.

[Mau08] G. Maurin, Courbes algébriques et équations multiplicatives. Math. Ann. 340 (2008), no. 4, 789-824. Zbl 1154.14017 MR 2407327

[Noo95] R. Noot, Abelian varieties - Galois representations and properties of ordinary reduction. Compositio Math. 97 (1995), 161-171. Zbl 0868.14021 MR 1355123

[Ogu82] A. Ogus, Hodge cycles and crystalline cohomology. In Hodge cycles, motives, and Shimura varieties, Lecture Notes in Math. 900. Springer-Verlag, Berlin 1982, 357-414. Zbl 0538.14010 MR 0654325

[Phi86] P. Philippon, Lemmes de zéros dans les groupes algébriques commutatifs. Bull. Soc. Math. France 114 (1986), no. 3, 355-383. Zbl 0617.14001 MR 0878242

[Phi95] P. Philippon, Sur des hauteurs alternatives III. J. Math. Pures Appl. (9) 74 (1995), no. 4, 345-365. Zbl 0878.11025 MR 1341770

[PW88] P. Philippon et M. Waldschmidt, Formes linéaires de logarithmes dans les groupes algébriques commutatifs III. Illinois J. Math. 32 (1988), no. 2, 281-314. Zbl 0651.10023 MR 0945864

[Pin98] R. Pink, $l$-adic algebraic monodromy groups, cocharacters, and the MumfordTate conjecture. J. Reine Angew. Math. 495 (1998), 187-237. Zbl 0920.14006 MR 1603865

[Pin05] R. Pink, A common generalization of the conjectures of André-Oort, Manin-Mumford and Mordell-Lang. Prépublication, 2005.

[Rat04] N. Ratazzi, Densité de points et minoration de hauteur. J. Number Theory 106 (2004), no. 1, 112-127. Zbl 1065.11049 MR 2049595

[Rat08] N. Ratazzi, Intersection de courbes et de sous-groupes et problèmes de minoration de hauteur dans les variétés abéliennes C.M. Ann. Inst. Fourier (Grenoble) 58 (2008), no. 5, 1575-1633. Zbl 1156.11025 MR 2445828

[RV03] G. Rémond et E. Viada, Problème de Mordell-Lang modulo certaines sous-variétés abéliennes. Internat. Math. Res. Notices 35 (2003), 1915-1931 Zbl 1072.11038 MR 1995142

[Ser68] J. P. Serre, Abelian l-adic representations and elliptic curves. Benjamin, New York, 1968. Zbl 0186.25701 MR 0263823 
[Via03] E. Viada, The intersection of a curve with algebraic subgroups in a product of elliptic curves. Ann. Sc. Norm. Super. Pisa Cl. Sci. (5) 2 (2003), no. 1, 47-75. Zbl 05019603 MR 1990974

[Via08a] E. Viada, The intersection of a curve with a union of translated codimension-two subgroups in a power of an elliptic curve. Algebra Number Theory 2 (2008), no. 3, 249-298. Zbl 1168.11024 MR 2407116

[Via08b] E. Viada, Non-dense subsets of algebraic points on a variety. Prépublication 2008, arXiv:0711.3533v3 [math.NT].

[Zha92] S. Zhang, Positive line bundles on arithmetic surfaces. Ann. of Math. 136 (1992), 569-587. Zbl 0788.14017 MR 1189866

[Zha95] S. Zhang, Positive line bundles on arithmetic varieties. J. Amer. Math. Soc. 8 (1995), 187-221. Zbl 0861.14018 MR 1254133

[Zi102] B. Zilber, Exponential sums equations and the Schanuel conjecture. J. London Math. Soc. (2) 65 (2002), no. 1, 27-44. Zbl 1030.11073 MR 1875133

Received July 30, 2008

Aurélien Galateau, Mathematisches Institut, Universität Basel, Rheinsprung 21, 4051 Basel, Switzerland, et Département de Mathématiques, Bâtiment 425, Faculté des Sciences d'Orsay, Université Paris-Sud 11, 91405 Orsay Cedex, France

E-mail: aurelien.galateau@math.u-psud.fr 